\title{
SETS WITH LARGE INTERSECTION PROPERTIES IN METRIC SPACES
}

\author{
FELIPE NEGREIRA, EMILIANO SEQUEIRA
}

\begin{abstract}
In this work we reproduce the characterization of $\mathcal{G}^{s}$-sets from the euclidean setting [12] to more general metric spaces. These sets have Hausdorff dimension at least $s$ and are closed by countable intersections, which is particularly useful to estimate the dimension of the so called sets of $\alpha$-approximable points (that typically appear in Diophantine approximations).
\end{abstract}

\section{INTRODUCTION}

In [12] Falconer gave a characterization for certain classes of sets in $\mathbb{R}^{d}$ with Hausdorff dimension at least $s$ that have a "large intersection" property in the sense that their countable intersections also have dimension $s$ or larger. These are the so called $\mathcal{G}^{s}$-sets: subsets $F \subset \mathbb{R}^{d}$ which are $G_{\delta}$ (countable intersection of open sets) and such that

$$
\operatorname{dim}_{\mathcal{H}} \bigcap_{i} f_{i}(F) \geqslant s
$$

holds for all sequences of similarity transformations $\left\{f_{i}\right\}$, where $\operatorname{dim}_{\mathcal{H}}$ denotes the Hausdorff dimension. Equivalently, one can describe this sets in terms of bi-Lipschitz functions: $F$ is a $\mathcal{G}^{s}$-set if and only if for every open set $V \subset \mathbb{R}^{d}$ and every sequence of bi-Lipschitz maps $f_{i}: V \rightarrow \mathbb{R}^{d}$

$$
\operatorname{dim}_{\mathcal{H}} \bigcap_{i} f_{i}^{-1}(F) \geqslant s
$$

Further, the results in [12] show that $\mathcal{G}^{s}$ is the maximal class of $G_{\delta}$ sets with Hausdorff dimension $s$ or larger, and which is closed under countable intersections and similarities or bi-Lipschitz homeomorphisms in general.

The main tool used by Falconer to describe $\mathcal{G}^{s}$-sets are the so called net measures and its respective contents. To construct such measures one needs a suitable family of sets (see [22, Definition 31]), which in $\mathbb{R}^{d}$ take the natural form of dyadic partitions: this is, cubes with the form $Q_{j, k}=2^{j}\left(k+[0,1)^{d}\right), j \in \mathbb{Z}, k \in \mathbb{Z}^{d}$. Using these dyadic cubes, the net (outer) measure $\mathcal{M}^{s}$ of a set $E \subset \mathbb{R}^{d}$ is defined by a Carathéodory construction as $\mathcal{M}^{s}(E):=\lim _{r \downarrow 0} \mathcal{M}_{r}^{s}(E)$, where

$$
\mathcal{M}_{r}^{s}(E):=\inf \left\{\sum_{n} \operatorname{diam}\left(Q_{n}\right)^{s}: E \subset \bigcup_{n} Q_{n}, Q_{n} \text { dyadic cube with } \operatorname{diam}\left(Q_{n}\right) \leqslant r\right\} .
$$

In $[12$, Theorem B] it is claimed that (1) is equivalent to

$$
\mathcal{M}_{\infty}^{s}(F \cap Q)=\mathcal{M}_{\infty}^{s}(Q), \quad \text { for all dyadic cube } Q
$$

Actually, this equivalence as stated is not always true: Example 6.2 below shows that (3) is strictly stronger than (1). However, as noted by Bugeaud in [6], this problem can be easily fixed by weakening (3) to

$$
\mathcal{M}_{\infty}^{t}(F \cap Q)=\mathcal{M}_{\infty}^{t}(Q), \quad \text { for all dyadic cube } Q \text { and all } t<s .
$$

2010 Mathematics Subject Classification. 11J83, 28A78.

Key words and phrases. Diophantine approximations, metric spaces, net measures. 
When trying to adapt these definitions to other metric spaces, the problem with (1) is that we may have very few similarities or bi-Lipschitz maps and so we might end with a weak condition that fails to preserve some fundamental properties of $\mathcal{G}^{s}$-sets in $\mathbb{R}^{d}$. Indeed, as Example 6.1 shows, it is possible to construct one of such metric spaces where there exists a large class of sets verifying (1) but, for example, which is not closed under countable intersections. Instead, provided that we have a dyadic-type partition, (3) or (4) give a better generalization.

The existence of dyadic-type cubes in more general settings such as spaces of homogeneous type (quasi-metric spaces with a doubling Borel measure) is known since at least the work of M. Christ [7] in 1990. More recently, T. Hytönen and A. Kairema [16] gave a sharper dyadic decomposition for any geometrically doubling metric space. One could then expect the results of Falconer [12] to be also valid in these metric (or quasi-metric) spaces. However, dyadic cubes in an arbitrary metric space as understood in $[7,16]$ are not exactly perfect cubes as in $\mathbb{R}^{d}$ but rather quasi-balls: sets trapped in between a ball $B$ and a scalar multiple $\mathbf{K} B$, see $\S 2.2$ below. Thus, in the more general case, we only have an approximate idea of the diameter of a dyadic cube. The problem with this is that, a priory, we do not know whether the function $Q \mapsto \operatorname{diam}(Q)^{s}$ is sub-additive or not -a property that plays key role in many of the proofs of [12].

When we have a measure with enough regularity, a way to circumvent this is to change $\operatorname{diam}(Q)^{s}$ in $(2)$ for the measure of $Q$ taken with an appropriate exponent. For example, in $\mathbb{R}^{d}$ for $0<s \leqslant d$ and $0<r \leqslant \infty$ if we set

$$
\mathcal{M}_{\mathcal{L}, r}^{s}(E):=\inf \left\{\sum_{n} \mathcal{L}\left(Q_{n}\right)^{s / d}: E \subset \bigcup_{n} Q_{n}, Q_{n} \text { dyadic cube with } \mathcal{L}\left(Q_{n}\right) \leqslant r\right\},
$$

where $\mathcal{L}$ denotes the usual Lebesgue measure, then $\mathcal{M}_{\mathcal{L}}^{s}=\lim _{r \downarrow 0} \mathcal{M}_{\mathcal{L}, r}^{s}$ and $\mathcal{M}_{\mathcal{L}, \infty}^{s}$ are scalar multiples of $\mathcal{M}^{s}$ and $\mathcal{M}_{\infty}^{s}$ respectively. Moreover, one could work with more general Borel measures $\mu$ instead of $\mathcal{L}$ and still be able to reproduce much of Falconer's results. Indeed, in [21] Persson shows how, keeping the same standard dyadic partition in $\mathbb{R}^{d}$ but working with an arbitrary non-atomic locally finite Borel measure $\mu$, one can define the equivalent $\mathcal{G}_{\mu}^{s}$-sets in a way such that many the same properties of [12] still hold.

Here, using the same notation as in [21], we translate these definitions into a metric space of homogeneous type.

Definition 1.1. Let $(X, \mu)$ be a metric space of homogeneous type. Let $d=\operatorname{dim}_{\mathcal{H}} X$, take $\mathcal{Q}$ a dyadic partition in $X$ and $0<s \leqslant d$. We say that a $G_{\delta}$ set $F \subset X$ is a $\mathcal{J}_{\mu}^{s}$-set if

$$
\mathcal{M}_{\mu, \infty}^{s}(F \cap Q)=\mathcal{M}_{\mu, \infty}^{s}(Q)
$$

holds for all $Q \in \mathcal{Q}$. We donote the class of $\mathcal{J}_{\mu}^{s}$-sets in $X$ by $\mathcal{J}_{\mu}^{s}(X)$ or simply $\mathcal{J}_{\mu}^{s}$.

We also define the class of $\mathcal{G}_{\mu}^{s}$-sets in $X$ by

$$
\mathcal{G}_{\mu}^{s}=\mathcal{G}_{\mu}^{s}(X):=\bigcap_{t<s} \mathcal{J}_{\mu}^{t}(X)
$$

Note that the condition that defines a $\mathcal{G}_{\mu}^{s}$-set in a general metric space $X$ is effectively the equivalent of (4) in $\mathbb{R}^{d}$. For both of these type of sets the corresponding equivalences of $[12$, Theorem B] still hold.

Theorem 1.2. Let $(X, \mu)$ be a metric space of homogeneous type with $d=\operatorname{dim}_{\mathcal{H}} X$. Take $F \subset X a G_{\delta}$ subset and $0<s \leqslant d$. Then the following statements are equivalent:

(i) $F$ is a $\mathcal{J}_{\mu}^{s}$-set, i.e. for all dyadic cubes $Q$ we have

$$
\mathcal{M}_{\mu, \infty}^{s}(F \cap Q)=\mathcal{M}_{\mu, \infty}^{s}(Q) .
$$

(ii) For all open sets $U$ we have

$$
\mathcal{M}_{\mu, \infty}^{s}(F \cap U)=\mathcal{M}_{\mu, \infty}^{s}(U) .
$$


(iii) There exists a constant $0<c \leqslant 1$ such that for all open sets $U$ we have

$$
\mathcal{M}_{\mu, \infty}^{s}(F \cap U) \geqslant c \mathcal{M}_{\mu, \infty}^{s}(U) .
$$

(iv) There exists a constant $0<c \leqslant 1$ such that for all dyadic cubes $Q$ we have

$$
\mathcal{M}_{\mu, \infty}^{s}(F \cap Q) \geqslant c \mathcal{M}_{\mu, \infty}^{s}(Q) .
$$

Moreover, the same is true for $\mathcal{G}_{\mu}^{s}$-sets with the corresponding adaptations.

These classes of sets also share some of the properties with their euclidean counterpart $[12$, Theorem C].

Theorem 1.3. Let $(X, \mu)$ be a metric space of homogeneous type with $d=\operatorname{dim}_{\mathcal{H}} X$ and take $0<s \leqslant d$.

(i) If $0<t \leqslant s$, then $\mathcal{J}_{\mu}^{s} \subset \mathcal{J}_{\mu}^{t}$.

(ii) If $F \subset E \subset X$ and $F \in \mathcal{J}_{\mu}^{s}$, then $E \in \mathcal{J}_{\mu}^{s}$.

(iii) If $X$ is complete, then $\mathcal{J}_{\mu}^{s}$ is closed by countable intersections.

The same properties hold for $\mathcal{G}_{\mu}^{s}$-sets.

If the measure $\mu$ has more regularity, in particular if it is Ahlfors regular (i.e. if $\mu(B(x, r)) \sim r^{d}$ for all ball $B(x, r)$ in $\left.X\right)$ then a couple of properties can be added.

Theorem 1.4. Let $(X, \mu)$ be an Ahlfors regular space of dimension $d$ and take $0<s \leqslant d$.

(i) If $F \in \mathcal{J}_{\mu}^{s}$, then $\mathcal{H}_{\infty}^{s}(F)>0$ and in particular $\operatorname{dim}_{\mathcal{H}} F \geqslant s$.

(ii) Let $(Y, \nu)$ be another Ahlfors regular space and $f: X \rightarrow Y$ a bi-Lipschitz homeomorphism. Then $F \in \mathcal{J}_{\mu}^{s}(X)$ if and only if $f(F) \in \mathcal{J}_{\nu}^{s}(Y)$.

The same properties hold for $\mathcal{G}_{\mu}^{s}$-sets.

Point $(i)$ of Theorem 1.3 in particular means that $\mathcal{J}_{\mu}^{s}(X) \subset \mathcal{G}_{\mu}^{s}(X)$. Additionally, the equivalence between $\mathcal{M}_{\infty}$ and $\mathcal{M}_{\mathcal{L}, \infty}$ in $\mathbb{R}^{d}$ implies $\mathcal{G}_{\mathcal{L}}^{s}\left(\mathbb{R}^{d}\right)=\mathcal{G}^{s}\left(\mathbb{R}^{d}\right)$, where as before $\mathcal{L}$ denotes the Lebesgue measure in $\mathbb{R}^{d}$. Altogether

$$
\mathcal{J}_{\mathcal{L}}^{s}\left(\mathbb{R}^{d}\right) \subset \mathcal{G}_{\mathcal{L}}^{s}\left(\mathbb{R}^{d}\right)=\mathcal{G}^{s}\left(\mathbb{R}^{d}\right) .
$$

We will see, again in Example 6.2, that the first inclusion is strict. Therefore, although $\mathcal{J}_{\mathcal{L}}^{s}\left(\mathbb{R}^{d}\right)$ is composed of sets with Hausdorff dimension at least $s$ (by $(i)$ of Theorem 1.4), and it is closed under countable intersections (by (iii) in Theorem 1.3) and by bi-Lipschitz maps (by (ii) of Theorem 1.4), it is not maximal among classes of sets with those conditions since $\mathcal{G}^{s}\left(\mathbb{R}^{d}\right)$ is strictly larger.

Classic examples of $\mathcal{G}^{s}$-sets in $\mathbb{R}$ are those obtained by Diophantine approximations. That is, for a fixed $\alpha>2$ we take the set $F_{\alpha} \subset \mathbb{R}$ of all real numbers $x$ such that

$$
\left|x-\frac{p}{q}\right| \leqslant q^{-\alpha}
$$

is verified for infinitely many rational numbers $p / q$. This set is in $\mathcal{G}^{1 / \alpha}$, see e.g. [11, Example 8.9]. In fact, even for a smaller set of rationals as the dyadic centers $p / q=k 2^{-j}$ we still obtain a $\mathcal{G}^{1 / \alpha}$-set. Points in this set have been called $\alpha$-approximable (by dyadics) and they are useful in the study of multifractal analysis of functions in Sobolev and Besov spaces $[2,13,17]$. There are several generalizations and applications of these classical Diophantine approximations, we refer to $[3,4,5,6,8,9]$ and the references therein.

Observe that the points $x \in F_{\alpha}$ can be expressed as the limit of a rational sequences $\left\{p_{j} / q_{j}\right\}$ where $0<q_{j}<q_{j+1}$ and $\left|x-p_{j} / q_{j}\right| \leqslant\left(q_{j}\right)^{-\alpha}$. This can be naturally generalized to any metric space by what we call $(\mathcal{E}, \alpha)$-approximable points (or simply $\alpha$-approximable points): limits of sequences $\left\{x_{j}\right\}$ that converge at speed $\epsilon_{j}^{\alpha}$, where $\mathcal{E}=\left\{\epsilon_{j}\right\}$ is a sequence 
converging to 0 and $\alpha$ is a real number bigger than 1 . For the precise definition see $\S 2.5$ below.

As an application of the previous results we show that $\alpha$-approximable points are particular cases of $\mathcal{G}_{\mu}^{s}$-sets in certain regular measurable metric spaces.

Theorem 1.5. Let $(X, \mu)$ a complete Ahlfors regular space of dimension d. Let $\alpha>1$ and $F \subset X$ a set of $(\mathcal{E}, \alpha)$-approximable points. Then $F \in \mathcal{G}_{\mu}^{d / \alpha}$ and therefore $\operatorname{dim}_{\mathcal{H}} F \geqslant d / \alpha$. Moreover, if the sequence $\mathcal{E}$ has exponential decay, then $\operatorname{dim}_{\mathcal{H}} F=d / \alpha$.

Let us remark that the lower bound for the Hausdorff dimension of these type of sets has been previously obtained on compact Ahlfors regular spaces in [4, Theorem 2] by using the concept of ubiquitous systems, first developed in [9].

The upper bound in Theorem 1.5, however, is not true in general in the sense that exponential decay is required. For example, Jarník's Theorem [18] shows that the Hausdorff dimension of the set of points obtained by Diophantine approximation on $\mathbb{R}$ for $\alpha>2$ (where the decay of convergence is polynomial) is actually $2 / \alpha$ (in fact, it is also possible to prove that it is a $\mathcal{G}^{2 / \alpha}$-set, see $\left.[11, \S 10]\right)$. In $\S 5$ we give an upper bound for the dimension in case of polynomial decay.

To end with, let us briefly describe how this work is organized. In Section 2 we give the basic definitions and properties on spaces of homogeneous type, along some preliminary results. In Sections 3 we prove Theorem 1.2 and in Section 4 we prove Theorems 1.3 and 1.4. In Section 5 we apply previous results to show that $\alpha$-approximable sets are a special case of $\mathcal{G}_{\mu}^{s}$-sets by proving Theorem 1.5 . Finally in Section 6 we compare $\mathcal{J}_{\mu}^{s}$-sets with $\mathcal{G}_{\mu}^{s}$-sets and their links with previous established definitions $[6,12]$.

\section{Preliminaries}

2.1. Doubling measures and doubling spaces. Let $X$ be a metric space. We denote the distance between two points $x, y \in X$ by $|x-y|$, and the ball of center $x \in X$ and radius $r>0$ by $B(x, r):=\{y:|y-x| \leqslant r\}$ (our balls will be always closed).

A Borel measure $\mu$ on $X$ is said to be doubling if it is finite and positive in every ball and there exists a constant $\mathbf{C} \geqslant 1$ such that for every $x \in X$ and $r>0$,

$$
\mu(B(x, 2 r)) \leqslant \mathbf{C} \mu(B(x, r)) .
$$

This inequality can be reformulated as

$$
\mu(B(x, R)) \leqslant \mathbf{C}\left(\frac{R}{r}\right)^{\beta} \mu(B(x, r))
$$

for any $0<r<R$ and $x \in X$, and where $\beta \geqslant 1$ is some independent constant (see e.g. $[15$, Lemma 4.7]). In this case we also say that $(X, \mu)$ is a metric space of homogeneous type.

A metric space $X$ is (geometrically) doubling if there exists a constant $N \in \mathbb{N}$ such that every bounded subset $A \subset X$ can be covered by no more than $N$ subsets with diameter at most $\operatorname{diam}(A) / 2$. A metric space of homogeneous type is always doubling. Reciprocally, every complete doubling space admits a doubling measure, see $[15, \S 13]$.

We can mimic the proof of Bolzano-Weierstrass property on $\mathbb{R}^{d}$ to get the following result:

Lemma 2.1. Let $X$ be a complete doubling metric space. Then every bounded and closed subset of $X$ is compact.

2.2. Dyadic decomposition. By a dyadic decomposition of parameter $0<\delta<1$ on a metric space $X$ we mean a family $\mathcal{Q}$ of measurable subsets $Q_{j, k}$, called dyadic cubes, with $j \in \mathbb{Z}$ and $k \in I_{j}$ satisfying the following properties: 
- For every $j \in \mathbb{Z}$

(6)

$$
X=\bigcup_{k \in I_{j}} Q_{j, k}
$$

- There is a uniform constant $\mathbf{K} \geqslant 1$ such that every dyadic cube $Q_{j, k}$ is a $\mathbf{K}$-quasiball with radius $\delta^{j}$. That is, there exists a point $x \in Q_{j, k}$ such that

$$
B\left(x, \delta^{j}\right) \subset Q_{j, k} \subset B\left(x, \mathbf{K} \delta^{j}\right) .
$$

- If $i \geqslant j$, then

$$
\text { either } Q_{i, k^{\prime}} \subset Q_{j, k} \text { or } Q_{i, k^{\prime}} \cap Q_{j, k}=\emptyset .
$$

We will say that the level of an arbitrary dyadic cube $Q$ is $j \in \mathbb{N}$, and $\operatorname{denote} \operatorname{lev}(Q)=j$, if there exists $k \in I_{j}$ such that $Q=Q_{j, k}$.

As said in the introduction, due to [16], for an appropriate choice of $\delta$ and $\mathbf{K}$ one can always construct a dyadic decomposition on a metric space of homogeneous type. Notice that, if $X$ is bounded, there exitst $j_{0} \in \mathbb{Z}$ such that $\# I_{j}=1$ and for every $j \leqslant j_{0}$ the dyadic cube of level $j$ is the whole space $X$.

2.3. Hausdorff and net outer measures. In a general metric space $X$ the Hausdorff outer measure for any $s \geqslant 0$ is defined in the following way: given $E \subset X$,

$$
\mathcal{H}^{s}(E):=\lim _{r \downarrow 0} \mathcal{H}_{r}^{s}(E), \quad \text { with } \mathcal{H}_{r}^{s}(E):=\inf \left\{\sum_{n} \operatorname{diam}\left(E_{n}\right)^{s}: E \subset \bigcup_{n} E_{n}, \operatorname{diam}\left(E_{n}\right) \leqslant r\right\} .
$$

The Hausdorff content of a set $E$ is $\mathcal{H}_{r}^{s}(E)$ for $r=\infty$. From this definition it follows that if $\mathcal{H}^{s}(E)<\infty$, then $\mathcal{H}^{t}(E)=0$ for every $t>s$. The Hausdorff dimension of a subset $E \subset X$ is then defined as

$$
\operatorname{dim}_{\mathcal{H}} E:=\inf \left\{s \geqslant 0: \mathcal{H}^{s}(E)=0\right\}=\sup \left\{s \geqslant 0: \mathcal{H}^{s}(E)=\infty\right\} .
$$

Using Carathéodory's Theorem for metric spaces one can see that $\mathcal{H}^{s}$ defines a Borel measure on $X$ for every $s \geqslant 0$.

Next, in a metric space of homogeneous type $X$ with a dyadic decomposition $\mathcal{Q}$ and Hausdorff dimension $d$ (which is always finite, see e.g. $[15, \S 10]$ ) we define a net outer measure for any $s \geqslant 0$ as $\mathcal{M}_{\mu}^{s}(E):=\lim _{r \downarrow 0} \mathcal{M}_{\mu, r}^{s}(E)$, where

$$
\mathcal{M}_{\mu, r}^{s}(E):=\inf \left\{\sum_{n} \mu\left(Q_{n}\right)^{s / d}: E \subset \bigcup_{n} Q_{n}, Q_{n} \text { is a dyadic cube with } \mu\left(Q_{n}\right) \leqslant r\right\} .
$$

We also define the net content of a subset $E \subset X$ as $\mathcal{M}_{\mu, r}^{s}(E)$ with $r=\infty$.

Different dyadic decomposition may give different net measures and contents but they are nonetheless equivalent:

Proposition 2.2. Let $s \geqslant 0$, and $\mathcal{Q}$ and $\widetilde{\mathcal{Q}}$ be two dyadic decompositions on a metric space of homogeneous type $(X, \mu)$ and $\mathcal{M}_{\mu}^{s}$ and $\widetilde{\mathcal{M}}_{\mu}^{s}$ their respective net measures. Then, for every $s \geqslant 0$ there exists a constant $L \geqslant 1$, such that

$$
L^{-1} \widetilde{\mathcal{M}}_{\mu}^{s} \leqslant \mathcal{M}_{\mu}^{s} \leqslant L \widetilde{\mathcal{M}}_{\mu}^{s} \text {. }
$$

The same is true for the (respective) contents.

Before proving Proposition 2.2 let us see an elementary lemma that will be also useful in the future. 
Lemma 2.3. Fix a dyadic decomposition $\left\{Q_{j, k}\right\}$ of parameter $\delta$ on a metric space of homogeneous type $(X, \mu)$. There exists an increasing function $H:(0,+\infty) \rightarrow(1,+\infty)$ such that the number of dyadic cubes of level $j$ that intersect a ball of radius $R$ is at most $H\left(R / \delta^{j}\right)$. Furthermore, if $Q$ and $Q^{\prime}$ are two of these cubes, then

$$
H\left(R / \delta^{j}\right)^{-1} \mu(Q) \leqslant \mu\left(Q^{\prime}\right) \leqslant H\left(R / \delta^{j}\right) \mu(Q) .
$$

Proof. Let $\mathbf{K}$ be the constant as in (7) and denote by $\left\{Q_{i}\right\}_{i \in I}$ the set of dyadic cubes of level $j$ that intersect a ball $B(x, R)$. Then the triangle inequality gives $Q_{i} \subset B^{\prime}:=$ $B\left(x, R+2 \mathbf{K} \delta^{j}\right)$ for every $i \in I$.

Now we consider a set of points $\left\{x_{i}\right\}_{i \in I}$ such that $B\left(x_{i}, \delta^{j}\right) \subset Q_{i}$. Then using (5) we have

$$
\begin{aligned}
\mu\left(B^{\prime}\right) \leqslant \mu\left(B\left(x_{i}, 2\left(R+2 \mathbf{K} \delta^{j}\right)\right)\right) & \leqslant \mathbf{C} 2^{\beta}\left(\frac{R+2 \mathbf{K} \delta^{j}}{\delta^{j}}\right)^{\beta} \mu\left(B\left(x_{i}, \delta^{j}\right)\right) \\
& \leqslant \mathbf{C} 2^{\beta}\left(\frac{R+2 \mathbf{K} \delta^{j}}{\delta^{j}}\right)^{\beta} \mu\left(Q_{i}\right) .
\end{aligned}
$$

Defining $H(t):=\mathbf{C} 2^{\beta}(t+2 \mathbf{K})^{\beta}$ we immediately get (10) since

$$
\mu\left(Q_{i}\right) \leqslant \mu\left(B^{\prime}\right) \leqslant H\left(R / \delta^{j}\right) \mu\left(Q_{i}\right) .
$$

Together with the fact that the cubes $\left\{Q_{i}\right\}_{i \in I}$ are disjoint this also yields

$$
\frac{\# I}{H\left(R / \delta^{j}\right)} \mu\left(B^{\prime}\right) \leqslant \sum_{i \in I} \mu\left(Q_{i}\right) \leqslant \mu\left(B^{\prime}\right)
$$

and as a consequence $\# I \leqslant H\left(R / \delta^{j}\right)$.

Observe that in Lemma 2.3 one can change the ball of radius $R$ for an arbitrary set of diameter $R$ and $H\left(R / \delta^{j}\right)$ still works as an upper bound.

Proof of Proposition 2.2. Let $\delta_{1}$ and $\delta_{2}$ the parameters of $\mathcal{Q}$ and $\widetilde{\mathcal{Q}}$, and $\mathbf{K}_{1}$ and $\mathbf{K}_{2}$ their quasi-ball constants as in (7). Fix a subset $E \subset X$ and $r \in(0,+\infty]$. We take $\left\{Q_{n}\right\}$ a covering of $E$ by dyadic cubes of $\mathcal{Q}_{1}$ whose measures do not exceed $r$ and denote $j_{n}=\operatorname{lev}\left(Q_{n}\right)$.

For every $n$ we take $\ell_{n} \in \mathbb{Z}$ such that $\delta_{2}^{\ell_{n}} \leqslant \delta_{1}^{j_{n}}<\delta_{2}^{\ell_{n}-1}$. Using Lemma 2.3 and the fact that $Q_{n}$ is included in a ball of radius $\mathbf{K}_{1} \delta_{1}^{j_{n}}$ we can cover $Q_{n}$ with no more than $H_{2}\left(\mathbf{K}_{1} \delta_{1}^{j_{n}} / \delta_{2}^{\ell_{n}}\right) \leqslant H_{2}\left(\mathbf{K}_{1} / \delta_{2}\right)$ cubes of level $\ell_{n}$ in $\widetilde{\mathcal{Q}}$, where $H_{2}$ is the increasing function given by Lemma 2.3 for the covering $\widetilde{\mathcal{Q}}$. We denote these cubes by $\left\{P_{n, i}\right\}$.

If $B\left(x_{n}, \delta_{1}^{j_{n}}\right) \subset Q_{n} \subset B\left(x_{n}, \mathbf{K}_{1} \delta^{j_{n}}\right)$ are the associated balls, then for every $i$ we get $P_{n, i} \subset B\left(x_{n}, \mathbf{K}_{1} \delta_{1}^{j_{n}}+2 \mathbf{K}_{2} \delta_{2}^{\ell_{n}}\right)$. Using (5) we have

$$
\begin{aligned}
\mu\left(P_{n, i}\right) & \leqslant \mu\left(B\left(x_{n}, \mathbf{K}_{1} \delta_{1}^{j_{n}}+2 \mathbf{K}_{2} \delta_{2}^{\ell_{n}}\right)\right) \\
& \leqslant \mathbf{C}\left(\frac{\mathbf{K}_{1} \delta_{1}^{j_{n}}+2 \mathbf{K}_{2} \delta_{2}^{\ell_{n}}}{\delta_{1}^{j_{n}}}\right)^{\beta} \mu\left(B\left(x_{n}, \delta_{1}^{j_{n}}\right)\right) \\
& \leqslant \mathbf{C}\left(\mathbf{K}_{1}+2 \mathbf{K}_{2}\right)^{\beta} \mu\left(Q_{n}\right) .
\end{aligned}
$$

This implies

$$
\sum_{n} \sum_{i} \mu\left(P_{n, i}\right)^{s / d} \leqslant \sum_{n} H_{2}\left(\mathbf{K}_{1} / \delta_{2}\right) \mathbf{C}^{s / d}\left(\mathbf{K}_{1}+2 \mathbf{K}_{2}\right)^{\beta s / d} \mu\left(Q_{n}\right)^{s / d} .
$$

Since $\mu\left(Q_{n}\right) \leqslant r$, by definition we have $\sum_{n, i} \mu\left(P_{n, i}\right)^{s / d} \geqslant \widetilde{\mathcal{M}}_{\mu, r^{\prime}}^{s}(E)$, where $r^{\prime}=\mathbf{C}\left(\mathbf{K}_{1}+2 \mathbf{K}_{2}\right)^{\beta} r$. Thus, taking the infimum over all $\mathcal{Q}_{1}$-dyadic decompositions we obtain

$$
\widetilde{\mathcal{M}}_{\mu, r^{\prime}}^{s}(E) \leqslant H_{2}\left(\mathbf{K}_{1} / \delta_{2}\right) \mathbf{C}^{s / d}\left(\mathbf{K}_{1}+2 \mathbf{K}_{2}\right)^{\beta s / d} \mathcal{M}_{\mu, r}^{s}(E) \text {. }
$$


If $r=+\infty$ this means that

$$
\widetilde{\mathcal{M}}_{\mu, \infty}^{s}(E) \leqslant H_{2}\left(\mathbf{K}_{1} / \delta_{2}\right) \mathbf{C}^{s / d}\left(\mathbf{K}_{1}+2 \mathbf{K}_{2}\right)^{\beta s / d} \mathcal{M}_{\mu, \infty}^{s}(E) .
$$

If not, we take limits when $r \rightarrow 0$ to obtain

$$
\widetilde{\mathcal{M}}_{\mu}^{s}(E) \leqslant H_{2}\left(\mathbf{K}_{1} / \delta_{2}\right) \mathbf{C}^{s / d}\left(\mathbf{K}_{1}+2 \mathbf{K}_{2}\right)^{\beta s / d} \mathcal{M}_{\mu}^{s}(E) .
$$

The reverse inequality follows symmetrically. Thus, if we define

$$
L:=\max \left\{H_{2}\left(\mathbf{K}_{1} / \delta_{2}\right) \mathbf{C}^{s / d}\left(\mathbf{K}_{1}+2 \mathbf{K}_{2}\right)^{\beta s / d}, H_{1}\left(\mathbf{K}_{2} / \delta_{1}\right) \mathbf{C}^{s / d}\left(\mathbf{K}_{2}+2 \mathbf{K}_{1}\right)^{\beta s / d}\right\} .
$$

the desired inequalities are verified.

We will be mostly working with the content $\mathcal{M}_{\mu, \infty}^{s}$. Note that, given $0<s \leqslant d$,

$$
\mathcal{M}_{\mu, \infty}^{s}(Q)=\mu(Q)^{s / d}
$$

holds for all dyadic cubes $Q$. This follows from the $\sigma$-additivity of $\mu$ and the sub-additivity of the function $x \mapsto x^{\tau}$ when $\tau \in(0,1]$.

2.4. Ahlfors regular spaces. An Ahlfors regular space is a metric space $X$ with a Borel measure $\mu$ for which there exist two constants $d>0$ and $\mathbf{A}>0$ such that

$$
\mathbf{A}^{-1} r^{d} \leqslant \mu(B(x, r)) \leqslant \mathbf{A} r^{d}
$$

holds for all $0<r \leqslant \operatorname{diam}(X)$ and $x \in X$. The constant $d$ is sometimes referred as the dimension of $X$ and indeed it coincides with the Hausdorff dimension as defined in $\S 2.3$. Moreover, it can be proved that $C^{-1} \mathcal{H}^{d} \leqslant \mu \leqslant C \mathcal{H}^{d}$ for some constant $C>0$, see e.g. $[15, \S 8.7]$. In fact, this translates to the equivalence between any Hausdorff measure and its net measure counterpart:

Lemma 2.4. Let $(X, \mu)$ be an Ahlfors regular metric space of dimension d, then given $0<s \leqslant d$ there exists a constant $\mathbf{C}_{0}>0$ such that

$$
\mathbf{C}_{0}^{-1} \mathcal{H}^{s}(E) \leqslant \mathcal{M}_{\mu}^{s}(E) \leqslant \mathbf{C}_{0} \mathcal{H}^{s}(E),
$$

holds for all $E \subset X$. The same inequality holds for the contents $\mathcal{M}_{\mu, \infty}^{s}$ and $\mathcal{H}_{\infty}^{s}$.

Proof. Let $\mathcal{Q}$ be a dyadic decomposition with parameter $\delta \in(0,1)$ and quasi-ball constant $\mathbf{K}$. By slightly modifying $\mathbf{A}$ in (12), we have that if $Q \in \mathcal{Q}$ has level $\ell$ with $\delta^{\ell+1} \leqslant \operatorname{diam}(X)$, then

$$
\begin{gathered}
\operatorname{diam}(Q) \leqslant 2 \mathbf{K} \delta^{\ell} \\
\mathbf{A}^{-1} \delta^{\ell d} \leqslant \mu(Q) \leqslant \mathbf{A} \mathbf{K}^{d} \delta^{\ell d} .
\end{gathered}
$$

Now take an arbitrary subset $E \subset X$ together with a dyadic covering $\left\{Q_{n}\right\}$ with $\mu\left(Q_{n}\right) \leqslant$ $r$ for all $n$. We can assume that $\delta^{\operatorname{lev}\left(Q_{n}\right)+1} \leqslant \operatorname{diam}(X)$ for every $n$. Using (13) and the lower bound of (14) we see that

$$
\operatorname{diam}\left(Q_{n}\right) \leqslant 2 \mathbf{K A}^{1 / d} \mu\left(Q_{n}\right)^{1 / d} \leqslant 2 \mathbf{K A}^{1 / d} r^{1 / d}
$$

holds for all $n$. Thus, if we set $r^{\prime}:=2 \mathbf{K A}^{1 / d} r^{1 / d}$ we have that

$$
\mathcal{H}_{r^{\prime}}^{s}(E) \leqslant \sum_{n} \operatorname{diam}\left(Q_{n}\right)^{s} \leqslant 2^{s} \mathbf{K}^{s} \mathbf{A}^{s / d} \sum_{n} \mu\left(Q_{n}\right)^{s / d} .
$$

Taking infimum among all dyadic coverings $\left\{Q_{n}\right\}$ and then letting $r \rightarrow 0$ we obtain $\mathcal{H}^{s}(E) \leqslant 2^{s} \mathbf{K}^{s} \mathbf{A}^{s / d} \mathcal{M}_{\mu}^{s}(E)$. If we fix $r=\infty$, then $\mathcal{H}_{\infty}^{s}(E) \leqslant 2^{s} \mathbf{K}^{s} \mathbf{A}^{s / d} \mathcal{M}_{\mu, \infty}^{s}(E)$.

Next, consider a covering $\left\{U_{n}\right\}$ of $E$ by sets with diameter smaller than $r$. For each $n$ let $\ell_{n} \in \mathbb{Z}$ such that

$$
\mathbf{K} \delta^{\ell_{n}} \leqslant \operatorname{diam}\left(U_{n}\right) \leqslant \mathbf{K} \delta^{\ell_{n}-1}
$$


and cover $U_{n}$ with dyadic cubes of $\mathcal{Q}$ with level $\ell_{n}$, denoted by $Q_{n, 1}, \ldots, Q_{n, m_{n}}$. Observe that, by Lemma $2.3, m_{n} \leqslant H(\mathbf{K} / \delta)$ for every $n$. Using (15) and the upper bound of (14) we have

$$
\mu\left(Q_{n, i}\right) \leqslant \mathbf{A} \operatorname{diam}\left(U_{n}\right)^{d} \leqslant \mathbf{A} r^{d}
$$

for all $n$ and $i=1, \ldots, m_{n}$. Denoting $r^{\prime \prime}=\mathbf{A} r^{d}$ we see that

$$
\mathcal{M}_{\mu, r^{\prime \prime}}^{s}(E) \leqslant \sum_{n} \sum_{i} \mu\left(Q_{n, i}\right)^{s / d} \leqslant H(\mathbf{K} / \delta) \sum_{n} \operatorname{diam}\left(U_{n}\right)^{s} .
$$

Taking infinum among the coverings $\left\{U_{n}\right\}$ and then letting $r \rightarrow 0$, we get $\mathcal{M}_{\mu}^{s}(E) \leqslant$ $H(\mathbf{K} / \delta) \mathcal{H}^{s}(E)$. If we fix $r=\infty$, then $\mathcal{M}_{\mu, \infty}^{s}(E) \leqslant H(\mathbf{K} / \delta) \mathcal{H}_{\infty}^{s}(E)$.

2.5. $\alpha$-approximable points. Let $X$ be a metric space and $\epsilon, c_{1}, C_{1}>0$. We say that a discrete subset $\left\{x_{k}\right\} \subset X$ is a $\left(c_{1}, C_{1}, \epsilon\right)$-net if

$$
\left|x_{k}-x_{k^{\prime}}\right|>2 c_{1} \epsilon \quad k \neq k^{\prime}, \quad \inf _{k}\left|x_{k}-x\right|<C_{1} \epsilon \quad \forall x \in X .
$$

The first condition says that the balls centered at points in $\left\{x_{k}\right\}$ with radius $c_{1} \epsilon$ are pairwise disjoint, and the second condition implies that the family of balls taken with the same centers but with radius $C_{1} \epsilon$ is a covering of $X$. Observe that if $C_{1} \geqslant 2 c_{1}$, then one can use Zorn's lemma to construct $\left(c_{1}, C_{1}, \epsilon\right)$-nets on $X$.

Let $\alpha>1$ and $\mathcal{E}=\left\{\epsilon_{j}\right\}$ be a positive sequence with $\epsilon_{j} \rightarrow 0$. For each $j$, let $\left\{x_{j, k}\right\}_{k}$ be a $\left(c_{1}, C_{1}, \epsilon_{j}\right)$-net, consider

$$
E_{j}:=\bigcup_{k} B\left(x_{j, k}, \epsilon_{j}^{\alpha}\right)
$$

and then define the set of $(\mathcal{E}, \alpha)$-approximable points as

$$
F:=\limsup E_{j}=\bigcap_{j} \bigcup_{j^{\prime} \geqslant j} E_{j^{\prime}} .
$$

If $X$ is doubling then $F$ can also be defined as the set of points $y \in X$ such that

$$
\left|x_{j, k}-y\right| \leqslant \epsilon_{j}^{\alpha}
$$

holds for infinitely many points $x_{j, k}$, which is the same condition defining Diophatine approximations.

\section{Characterization of $\mathcal{J}_{\mu}^{s}$-Sets}

We begin by proving the equivalence provided by Theorem 1.2. Throughout this section we will assume that $(X, \mu)$ is a metric space of homogeneous type and that $\mathbf{C}>0, \beta>0$ are constants that satisfy (5). Further, we will take a dyadic decomposition $\mathcal{Q}$ with parameter $\delta \in(0,1)$ and quasi-ball constant $\mathbf{K}$ as in $(7)$.

Proof of Theorem 1.2. Observe that once the theorem is proved for $\mathcal{J}_{\mu}^{s}$-sets, it automatically follows for $\mathcal{G}_{\mu}^{s}$-sets. Let us then prove the equivalences for $\mathcal{J}_{\mu}^{s}$-sets.

Note that $(i i) \rightarrow($ iii $)$ is immediate by taking $c=1$. The direction $(i i i) \rightarrow(i v)$ follows from the fact that

$$
\mathcal{M}_{\mu, \infty}^{s}(\operatorname{int}(Q)) \geqslant c_{0} \mathcal{M}_{\mu, \infty}^{s}(Q)
$$

for all dyadic cubes $Q$, where $c_{0}>0$ is an independent constant. Indeed, if (16) holds for all $Q$, then for every $F$ verifying (iii) we can find a constant $c>0$ such that

$$
\mathcal{M}_{\mu, \infty}^{s}(F \cap Q) \geqslant \mathcal{M}_{\mu, \infty}^{s}(F \cap \operatorname{int}(Q)) \geqslant c \mathcal{M}_{\mu, \infty}^{s}(\operatorname{int}(Q)) \geqslant c_{0} c \mathcal{M}_{\mu, \infty}^{s}(Q)
$$


for all dyadic cubes $Q$. Now, to see (16) note that if $j$ is the level of $Q$ and $\left\{Q_{n}\right\}$ is a family of dyadic cubes covering $\operatorname{int}(Q)$, then

$$
\begin{aligned}
\sum_{n} \mu\left(Q_{n}\right)^{s / d} \geqslant \mu(\operatorname{int}(Q))^{s / d} & \geqslant \mu\left(B\left(x_{Q}, \delta^{j} / 2\right)\right)^{s / d} \\
& \geqslant \mathbf{C}^{-s / d}(2 \mathbf{K})^{-\beta s / d} \mu\left(B\left(x_{Q}, \mathbf{K} \delta^{j}\right)\right)^{s / d} \\
& \geqslant \mathbf{C}^{-s / d}(2 \mathbf{K})^{-\beta s / d} \mu(Q)^{s / d} \\
& \geqslant \mathbf{C}^{-s / d}(2 \mathbf{K})^{-\beta s / d} \mathcal{M}_{\mu, \infty}^{s}(Q),
\end{aligned}
$$

where $x_{Q} \in Q$ verifies (7) and where we have also used (5) in the third inequality and (11) in the last one. Finally, (16) follows by taking $c_{0}=\mathbf{C}^{-s}(2 \mathbf{K})^{-\beta s}$.

Let us show $(i) \rightarrow(i i)$. Here we essentially reproduce [12, Lemma 1] to our current setting. Assume that $F \subset X$ verifies $(i)$ and take an open subset $U \subset X$. We need to show that for every arbitrary dyadic covering $\left\{P_{m}\right\}$ of $F \cap U$ we have

$$
\sum_{m} \mu\left(P_{m}\right)^{s / d} \geqslant \mathcal{M}_{\mu, \infty}^{s}(U) .
$$

First, due to (8), we may assume that $\left\{P_{m}\right\}$ is disjoint. Second, since $U$ is we can write $U=\bigcup_{n} Q_{n}$ where $\left\{Q_{n}\right\}$ is a family of disjoint dyadic cubes. Thus

$$
\bigcup_{n} F \cap Q_{n} \subset \bigcup_{m} P_{m}
$$

From here, and since $F \cap Q \neq \emptyset$ for any dyadic cube $Q$, we may assume, using the nested property (8), that for each $Q_{n}$ one (and only one) of the following is true:

(a) there exists a unique $m$ such that

$$
Q_{n} \subset P_{m},
$$

(b) $Q_{n}$ is not included in any $P_{m}$ and instead

$$
F \cap Q_{n} \subset \bigcup_{P_{m} \subset Q_{n}} P_{m}
$$

If $Q_{n}$ verifies (a), then clearly

$$
\sum_{P_{m} \cap Q_{n} \neq \emptyset} \mu\left(P_{m}\right)^{s / d} \geqslant \mu\left(Q_{n}\right)^{s / d}
$$

If $Q_{n}$ verifies (b), then

$$
\sum_{P_{m} \cap Q_{n} \neq \emptyset} \mu\left(P_{m}\right)^{s / d} \geqslant \mathcal{M}_{\mu, \infty}^{s}\left(F \cap Q_{n}\right)=\mathcal{M}_{\mu, \infty}^{s}\left(Q_{n}\right)=\mu\left(Q_{n}\right)^{s / d},
$$

since we are assuming that $F$ verifies $(i)$ and by also using (11) in the last equality. Thus, changing all those $P_{m}$ that are proper subcubes of some $Q_{n}$ by one copy of $Q_{n}$, and keeping the rest of the cubes $P_{m}$, we obtain a dyadic covering $\left\{K_{l}\right\}$ of $U$ such that

$$
\sum_{m} \mu\left(P_{m}\right)^{s / d} \geqslant \sum_{l} \mu\left(K_{l}\right)^{s / d} \geqslant \mathcal{M}_{\mu, \infty}^{s}(U)
$$

which gives us exactly what we wanted to prove.

Finally, let us show that $(i v) \rightarrow(i)$. Here we adapt the ideas of [14, Lemma 2]. Assume that $F$ verifies $(i v)$ and let $Q$ be an arbitrary dyadic cube. We want to show that for any arbitrary dyadic covering $\left\{Q_{n}\right\}$ of $F \cap Q$ we have

$$
\sum_{n} \mu\left(Q_{n}\right)^{s / d} \geqslant \mathcal{M}_{\mu, \infty}^{s}(Q) .
$$


As before, we can suppose that this covering is disjoint and further that $Q_{n} \subset Q$ for all $n$. Moreover, since $\mathcal{M}_{\mu, \infty}^{s}(F \cap Q)$ is finite (e.g. it can be covered by just $Q$ ) we may assume that

$$
\sum_{n} \mu\left(Q_{n}\right)^{s / d}<\infty
$$

Next, as the series is convergent, we can reorganize the sum according to the level of each cube: for each $j \geqslant \operatorname{lev}(Q)$, denote $L_{j}:=\left\{n: \operatorname{lev}\left(Q_{n}\right)=j\right\}$ so that

$$
\sum_{n} \mu\left(Q_{n}\right)^{s / d}=\sum_{j} \sum_{n \in L_{j}} \mu\left(Q_{n}\right)^{s / d} .
$$

Moreover, since $Q_{n} \subset Q$ for all $n$ and the subcubes are all disjoint, then an application of Lemma 2.3 shows that $L_{j}$ is finite for any $j$. Altogether this means that, given $\epsilon>0$, we can find $j_{0}$ such that

$$
\sum_{j \geqslant j_{0}} \sum_{n \in L_{j}} \mu\left(Q_{n}\right)^{s / d}<\epsilon .
$$

We then construct the following dyadic partition of $Q$ : let $\left\{P_{m}\right\}$ such that for each $m$ either

(c) $P_{m}=Q_{n}$ for some $n$ and $\operatorname{lev}\left(P_{m}\right)<j_{0}$,

(d) $\operatorname{lev}\left(P_{m}\right)=j_{0}$ and all the $Q_{n}$ that intersect $F \cap P_{m}$ are contained in $P_{m}$.

(This can be done from a $j_{0}$-level partition of dyadic subcubes of $Q$.) Let us first take $m_{0}$ such that $P_{m_{0}}$ satisfies (c) so that

$$
\sum_{Q_{n} \subset P_{m_{0}}} \mu\left(Q_{n}\right)^{s / d}=\mu\left(P_{m_{0}}\right)^{s / d} .
$$

In particular this means that

$$
\sum_{j<j_{0}} \sum_{n \in L_{j}} \mu\left(Q_{n}\right)^{s / d}=\sum_{\operatorname{lev}\left(P_{m}\right)<j_{0}} \mu\left(P_{m}\right)^{s / d} .
$$

If now $P_{m_{0}}$ satisfies (d) we then have that

$$
\sum_{Q_{n} \subset P_{m_{0}}} \mu\left(Q_{n}\right)^{s / d} \geqslant \mathcal{M}_{\mu, \infty}^{s}\left(F \cap P_{m_{0}}\right) \geqslant c \mathcal{M}_{\mu, \infty}^{s}\left(P_{m_{0}}\right)=c \mu\left(P_{m_{0}}\right)^{s / d}
$$

by using the fact that $F$ verifies $(i v)$ in the last inequality. This gives

$$
\sum_{j \geqslant j_{0}} \sum_{n \in L_{j}} \mu\left(Q_{n}\right)^{s / d} \geqslant c \sum_{\operatorname{lev}\left(P_{m}\right)=j_{0}} \mu\left(P_{m}\right)^{s / d} .
$$

Altogether (17), (18) and (19) yield

$$
\begin{aligned}
\sum_{j} \sum_{n \in L_{j}} \mu\left(Q_{n}\right)^{s / d} & =\sum_{j<j_{0}} \sum_{n \in L_{j}} \mu\left(Q_{n}\right)^{s / d}+\sum_{j \geqslant j_{0}} \sum_{n \in L_{j}} \mu\left(Q_{n}\right)^{s / d} \\
& =\sum_{\operatorname{lev}\left(P_{m}\right)<j_{0}} \mu\left(P_{m}\right)^{s / d}+\left(c^{-1}+1-c^{-1}\right) \sum_{j \geqslant j_{0}} \sum_{n \in L_{j}} \mu\left(Q_{n}\right)^{s / d} \\
& \geqslant \sum_{\operatorname{lev}\left(P_{m}\right)<j_{0}} \mu\left(P_{m}\right)^{s / d}+\sum_{\operatorname{lev}\left(P_{m}\right)=j_{0}} \mu\left(P_{m}\right)^{s / d}+\left(1-c^{-1}\right) \epsilon \\
& \geqslant \mathcal{M}_{\mu, \infty}^{s}(Q)+\left(1-c^{-1}\right) \epsilon,
\end{aligned}
$$

by also using that $\left\{P_{m}\right\}$ is a dyadic partition of $Q$ with cubes of level at most $j_{0}$ in the last inequality. Finally, since $\epsilon>0$ is arbitrary we conclude that $\sum_{n} \mu\left(Q_{n}\right)^{s / d} \geqslant \mathcal{M}_{\mu, \infty}^{s}(Q)$. 
To end this section let us note that characterizations (iii) or (iv) of Theorem 1.2 together with Proposition 2.2 show that Definition 1.1 is independent from the chosen dyadic partition.

\section{Properties of $\mathcal{J}_{\mu}^{s}$-Sets}

We begin by showing different properties of the classes $\mathcal{J}_{\mu}^{s}$ and $\mathcal{G}_{\mu}^{s}$ provided by Theorem 1.3. Here we take the same assumptions and use the same notation than in the previous section.

Proof of Theorem 1.3. As in Theorem 1.2 it is enough to show the results for the class $\mathcal{J}_{\mu}^{s}$.

( $i$ ) If $t \leqslant s$, then for all families of dyadic cubes $\left\{Q_{n}\right\}$ we have

$$
\sum_{n} \mu\left(Q_{n}\right)^{t / d} \geqslant\left(\sum_{n} \mu\left(Q_{n}\right)^{s / d}\right)^{t / s},
$$

which yields $\mathcal{M}_{\mu, \infty}^{t}(E) \geqslant\left(\mathcal{M}_{\mu, \infty}^{s}(E)\right)^{t / s}$ for all subset $E \subset X$. Together with (11) this implies $\mathcal{J}_{\mu}^{s} \subset \mathcal{J}_{\mu}^{t}$.

(ii) This is obvious from the definition of $\mathcal{J}_{\mu}^{s}$-set and the fact that $\mathcal{M}_{\mu}^{s}$ is an outer measure.

(iii) Here we adapt Lemma 4 of [12]. Let $\left\{F_{j}\right\}_{j \geqslant 1}$ be a sequence of sets belonging to the class $\mathcal{J}_{\mu}^{s}$. We will prove that $\bigcap_{j} F_{j} \in \mathcal{J}_{\mu}^{s}$.

We may assume that $\left\{F_{j}\right\}_{j \geqslant 1}$ is a decreasing sequence of open sets. To see this suppose that $\left\{F_{j}\right\}_{j \geqslant 1}$ is arbitrary and use the $G_{\delta}$ property to write each $F_{j}$ as the countable intersection of open sets. By $(i i)$ of this Theorem we know that each of those open sets must belong to $\mathcal{J}_{\mu}^{s}$, and due to $(i i)$ of Theorem 1.2 the finite intersection of open $\mathcal{J}_{\mu}^{s}$-sets belongs to $\mathcal{J}_{\mu}^{s}$. Then a diagonal argument allows us to write $\bigcap_{j \geqslant 1} F_{j}$ as the decreasing intersection of open $\mathcal{J}_{\mu}^{s}$-sets.

Next, take an open subset $U \subset X$. Suppose first that $U$ is bounded and let $\epsilon>0$. We will construct a decreasing sequence of open sets $\left\{U_{j}\right\}_{j \geqslant 1}$ such that for each $j \geqslant 1$

$$
\begin{aligned}
\overline{U_{j}} & \subset F_{j} \cap U \\
\mathcal{M}_{\mu, \infty}^{s}\left(U_{j}\right) & >\mathcal{M}_{\mu, \infty}^{s}(U)-\epsilon .
\end{aligned}
$$

To that end, given a sequence of positive real numbers $\left\{r_{j}\right\}$ we set

$$
U_{0}=U, \quad U_{j}=\left(F_{j} \cap U_{j-1}\right)_{\left(-r_{j}\right)} \text { for } j \geqslant 1,
$$

where for any given subset $S \subset X$ and $r>0$ we denote $S_{(-r)}:=\left\{x \in S: \inf _{y \notin S}|x-y|>\right.$ $r\}$ (which is always open). Note first $U_{j}$ verifies (20) for any $j \geqslant 1$. Secondly, we claim that if the sequence $\left\{r_{j}\right\}$ is chosen appropriately, then (21) is also met. Indeed if $U_{j-1}$ verifies (21), then $(i i)$ of Theorem 1.2 applied to $F_{j}$ gives

$$
\mathcal{M}_{\mu, \infty}^{s}\left(F_{j} \cap U_{j-1}\right)=\mathcal{M}_{\mu, \infty}^{s}\left(U_{j-1}\right)>\mathcal{M}_{\mu, \infty}^{s}(U)-\epsilon .
$$

Finally, as $\left(F_{j} \cap U_{j-1}\right)_{(-r)} \nearrow F_{j} \cap U_{j-1}$ when $r \searrow 0$, the Increasing Sets Lemma for net measures [22, Theorem 52] assures the existence of a small enough $r_{j}>0$ such that $U_{j}=\left(F_{j} \cap U_{j-1}\right)_{\left(-r_{j}\right)}$ verifies $(21)$.

Using (20) we see that if $\left\{Q_{n}\right\}$ is a dyadic covering of $\bigcap_{j \geqslant 1} F_{j} \cap U$ then

$$
\bigcap_{j \geqslant 1} \bar{U}_{j} \subset \bigcup_{n} Q_{n} \subset \bigcup_{n} B\left(x_{n}, \mathbf{K} \delta^{j_{n}}\right),
$$

where $j_{n}$ denotes the level of $Q_{n}$ and $B\left(x_{n}, \mathbf{K} \delta^{j_{n}}\right)$ is the ball on the right hand side of (7). Since $X$ is complete and $\overline{U_{j}}$ is closed and bounded for all $j$, then Lemma 2.1 implies that it must be compact. Further, since the sequence $\left\{\bar{U}_{j}\right\}$ is decreasing, there exits 
$j_{1} \geqslant 1$ for which $\bar{U}_{j_{1}} \subset \bigcup_{n} B\left(x_{n}, \mathbf{K} \delta^{j_{n}}\right)$. Finally, for each $n$ let $\left\{Q_{n, m}\right\}_{m}$ the family of cubes with level $j_{n}$ that intersect the ball $B\left(x_{n}, \mathbf{K} \delta^{j_{n}}\right)$ (which clearly contains $Q_{n}$ ). Thus $\bar{U}_{j_{1}} \subset \bigcup_{n} \bigcup_{m} Q_{n, m}$. In particular this means that

$$
\sum_{n} \sum_{m} \mu\left(Q_{n, m}\right)^{s / d} \geqslant \mathcal{M}_{\mu, \infty}^{s}\left(U_{j_{1}}\right) .
$$

Now, we apply Lemma 2.3 by comparing each $Q_{n, m}$ to $Q_{n}$ to obtain

$$
\sum_{n} \sum_{m} \mu\left(Q_{n, m}\right)^{s / d} \leqslant \sum_{n} H\left(\mathbf{K} \delta^{j_{n}} / \delta^{j_{n}}\right)^{s / d} \mu\left(Q_{n}\right)^{s / d} \leqslant H(\mathbf{K})^{s / d} \sum_{n} \mu\left(Q_{n}\right)^{s / d}
$$

Altogether, taking $c=H(\mathbf{K})^{-s}$ and using (21), the previous inequalities read

$$
\sum_{n} \mu\left(Q_{n}\right)^{s / d} \geqslant c \mathcal{M}_{\mu, \infty}^{s}\left(U_{j}\right)>c\left(\mathcal{M}_{\mu, \infty}^{s}(U)-\epsilon\right) .
$$

Finally, since $\left\{Q_{n}\right\}$ and $\epsilon>0$ are arbitrary we get

$$
\mathcal{M}_{\mu, \infty}^{s}\left(\bigcap_{j \geqslant 1} F_{j} \cap U\right) \geqslant c \mathcal{M}_{\mu, \infty}^{s}(U),
$$

If $U$ is unbounded one can use again the Increasing Sets Lemma to obtain the same inequality. Therefore, in any case, (iii) of Theorem 1.2 is verified for $\bigcap_{j \geqslant 1} F_{j}$ and so $\bigcap_{j \geqslant 1} F_{j} \in \mathcal{J}_{\mu}^{s}$.

We now proceed to prove Theorem 1.4. Here we are assuming in addition that $(X, \mu)$ is Ahlfors regular with dimension $d$.

Proof of Theorem 1.4. (i) Using Lemma 2.4 we have that if $F \in \mathcal{J}_{\mu}^{s}$, then

$$
\begin{aligned}
\mathcal{H}_{\infty}^{s}(F) \geqslant \mathbf{C}_{0}^{-1} \mathcal{M}_{\mu, \infty}^{s}(F) & \geqslant \mathbf{C}_{0}^{-1} \mathcal{M}_{\mu, \infty}^{s}(F \cap Q) \\
& \geqslant \mathbf{C}_{0}^{-1} \mathcal{M}_{\mu, \infty}^{s}(Q)=\mathbf{C}_{0}^{-1} \mu(Q)^{s / d}>0
\end{aligned}
$$

by using $(i)$ of Theorem 1.2 in the third equality for an arbitrary dyadic cube $Q$.

(ii) Suppose that $F \in \mathcal{J}_{\mu}^{\mathcal{S}}(X)$. By (iii) of Theorem 1.2 and Lemma 2.4, to prove that $f(F) \in \mathcal{J}_{\nu}^{s}(Y)$ it is enough to see that

$$
\mathcal{H}_{Y, \infty}^{s}(f(F) \cap U) \geqslant c \mathcal{H}_{Y, \infty}^{s}(U)
$$

holds for some constant $c>0$ and every open set $U \subset Y$.

Since $f$ is bi-Lipschitz, there exists $c_{f}>0$ such that

$$
c_{f}^{-1} \mathcal{H}_{Y, \infty}^{s}(f(E)) \leqslant \mathcal{H}_{X, \infty}^{s}(E) \leqslant c_{f} \mathcal{H}_{Y, \infty}^{s}(f(E))
$$

holds for every subset $E \subset X$. Then, using again (iii) of Theorem 1.2 and Lemma 2.4, we have

$$
\begin{aligned}
\mathcal{H}_{Y, \infty}^{s}(f(F) \cap U) & \geqslant c_{f}^{-1} \mathcal{H}_{X, \infty}^{s}\left(F \cap f^{-1}(U)\right) \\
& \geqslant \mathbf{C}_{1}^{-1} c_{f}^{-1} \mathcal{M}_{\mu}^{s}\left(F \cap f^{-1}(U)\right) \\
& \geqslant \mathbf{C}_{1}^{-1} c_{f}^{-1} \mathcal{M}_{\mu}^{s}\left(f^{-1}(U)\right) \\
& \geqslant \mathbf{C}_{1}^{-2} c_{f}^{-1} \mathcal{H}_{X, \infty}^{s}\left(f^{-1}(U)\right) \\
& \geqslant \mathbf{C}_{1}^{-2} c_{f}^{-2} \mathcal{H}_{Y, \infty}^{s}(U)
\end{aligned}
$$

where $\mathbf{C}_{1}$ is the maximum of the $\mathbf{C}_{0}$ 's of Lemma 2.4 applied for $X$ and $Y$, and where $U \subset Y$ is an arbitrary open set. The other implication is analogous taking the function $f^{-1}$. 
Note that for the proof of $(i)$ we only use the right hand side of the inequality in Lemma 2.4 and therefore the result applies to any space $X$ that verifies the right hand side of (12). In fact, in [21] this hypothesis is assumed to estimate dimensions of different types of sets.

\section{5. $\alpha$-APPROXIMABLE POINTS}

Throughout this section we work with the same construction laid out in $\S 2.5$ : let $\alpha>1$, and for each $j$ let $\left\{x_{j, k}\right\}_{k}$ be a $\left(c_{1}, C_{1}, \epsilon_{j}\right)$-net, where $\epsilon_{j} \rightarrow 0$, and set

$$
E_{j}:=\bigcup_{k} B\left(x_{j, k}, \epsilon_{j}\right), \quad F:=\lim \sup E_{j} .
$$

The following property of approxiamble points is key in the first part of Theorem 1.5. It uses the strategies of [11, Example 8.9].

Lemma 5.1. For every $0<t<d / \alpha$ there exists a constant $c>0$ such that

$$
\limsup _{j \rightarrow \infty} \mathcal{M}_{\mu, \infty}^{t}\left(Q \cap E_{j}\right) \geqslant c \mathcal{M}_{\mu, \infty}^{t}(Q),
$$

for every dyadic cube $Q$.

In order to prove the previous lemma we will need the following result.

Lemma 5.2. Let $D$ be a $K$-quasi-ball of radius $R \leqslant \operatorname{diam}(X)$. Consider

$$
\begin{gathered}
\mathcal{F}_{j}(D):=\left\{k: B\left(x_{j, k}, c_{1} \epsilon_{j}\right) \subset D\right\}, \\
\mathcal{F}^{j}(D):=\left\{k: B\left(x_{j, k}, c_{1} \epsilon_{j}\right) \cap D \neq \emptyset\right\},
\end{gathered}
$$

and let $n_{j}(D)=\# \mathcal{F}_{j}(D), m_{j}(D)=\# \mathcal{F}^{j}(D)$. Then there exist $j_{0}$ and a constant $C_{2} \geqslant 1$ such that

$$
C_{2}^{-1} \epsilon_{j}^{-d}\left(R-\left(c_{1}+C_{1}\right) \epsilon_{j}\right)^{d} \leqslant n_{j}(D) \leqslant m_{j}(D) \leqslant C_{2} \epsilon_{j}^{-d}\left(K R+2 c_{1} \epsilon_{j}\right)^{d}
$$

holds for all $j \geqslant j_{0}$.

Proof. Let $x_{0} \in X$ such that

$$
B\left(x_{0}, R\right) \subset D \subset B\left(x_{0}, K R\right) .
$$

To begin with, since $\epsilon_{j} \rightarrow 0$, we can take $j_{0}$ large enough so that $R-\left(c_{1}+C_{1}\right) \epsilon_{j}>0$ for all $j \geqslant j_{0}$. Remember that the family of balls $B\left(x_{j, k}, C_{1} \epsilon_{j}\right)$ covers $X$ and observe that if $k \notin \mathcal{F}_{j}(D)$ then the triangle inequality and the left hand side of (23) reads $B\left(x_{j, k}, C_{1} \epsilon_{j}\right) \cap$ $B\left(x_{0}, R-\left(c_{1}+C_{1}\right) \epsilon_{j}\right)=\emptyset$. Altogether this implies

$$
B\left(x_{0}, R-\left(c_{1}+C_{1}\right) \epsilon_{j}\right) \subset \bigcup_{k \in \mathcal{F}_{j}(D)} B\left(x_{j, k}, C_{1} \epsilon_{j}\right) .
$$

Then, using (12) we have

$$
\mathbf{A}^{-1}\left(R-\left(c_{1}+C_{1}\right) \epsilon_{j}\right)^{d} \leqslant \mu\left(B\left(x_{0}, R-\left(c_{1}+C_{1}\right) \epsilon_{j}\right)\right) \leqslant \sum_{k \in \mathcal{F}_{j}(D)} \mu\left(B\left(x_{j, k}, C_{1} \epsilon_{j}\right)\right) \leqslant n_{j}(D) \mathbf{A} C_{1}^{d} \epsilon_{j}^{d},
$$

from where

$$
\mathbf{A}^{-2} C_{1}^{-d} \epsilon_{j}^{-d}\left(R-\left(c_{1}+C_{1}\right) \epsilon_{j}\right)^{d} \leqslant n_{j}(D) .
$$

For the last inequality of (22) note that the balls $B\left(x_{j, k}, c_{1} \epsilon_{j}\right)$ with $k \in \mathcal{F}^{j}(D)$ are all included in $B\left(x_{0}, K R+2 c_{1} \epsilon_{j}\right)$, cf. (23). And since $\left\{x_{j, k}\right\}_{k}$ is a $\left(c_{1}, C_{1}, \epsilon_{j}\right)$-net, then those balls are pairwise disjoint. This yields

$$
m_{j}(D) \mathbf{A}^{-1} c_{1}^{d} \epsilon_{j}^{d} \leqslant \sum_{k \in \mathcal{F}^{j}(D)} \mu\left(B\left(x_{j, k}, c_{1} \epsilon_{j}\right)\right) \leqslant \mu\left(B\left(x_{0}, K R+2 c_{1} \epsilon_{j}\right)\right) \leqslant \mathbf{A}\left(K R+2 c_{1} \epsilon_{j}\right)^{d} .
$$

This proof finishes by taking $C_{2}=\max \left\{\mathbf{A}^{2} c_{1}^{-d}, \mathbf{A}^{2} C_{1}^{d}\right\}$. 
Now we prove Lemma 5.1.

Proof of Lemma 5.1. Let $Q$ be an arbitrary dyadic cube of level $\ell, R=\min \left\{\delta^{\ell}\right.$, $\left.\operatorname{diam}(X)\right\}$ and $x_{0} \in Q$ such that

$$
B\left(x_{0}, R\right) \subset Q \subset B\left(x_{0}, \mathbf{K} R\right) .
$$

From now on we fix $j_{0} \in \mathbb{N}$ so that (22) is verified for $j \geqslant j_{0}$ when we put $D=Q$ and $K=\mathbf{K}$. Furthermore, since $\epsilon_{j} \rightarrow 0$ when $j \rightarrow \infty$ and $\alpha>1$, we may also assume that $\epsilon_{j}^{\alpha} \leqslant \frac{1}{2} c_{1} \epsilon_{j}$ and $\left(c_{1}+C_{1}\right) \epsilon_{j} \leqslant \frac{1}{2} R$ for all $j \geqslant j_{0}$. For each $j \geqslant j_{0}$ we consider $\nu_{j}$ the probability measure that distributes the mass among the balls of the family

$$
\mathcal{S}_{j}(Q)=\left\{B\left(x_{j, k}, \epsilon_{j}^{\alpha}\right): k \in \mathcal{F}_{j}(Q)\right\} .
$$

That is

$$
\nu_{j}(A \cap B)=\frac{\mu(A \cap B)}{\mu(B) n_{j}(Q)}
$$

for every $B \in \mathcal{S}_{j}(Q)$.

Claim: Let $\varepsilon>0$. For every $j \geqslant j_{0}$ there exists $\left\{Q_{n}\right\}$ a dyadic covering of $Q \cap E_{j}$ that satisfies

$$
\sum_{n} \mu\left(Q_{n}\right)^{t / d} \leqslant H(2 \mathbf{K} / \delta)\left(\mathcal{M}_{\mu, \infty}^{t}\left(Q \cap E_{j}\right)+\varepsilon\right)
$$

and for each $\ell_{n}=\operatorname{lev}\left(Q_{n}\right)$ either

(a) $2 \mathbf{K} \delta^{\ell_{n}} \geqslant c_{1} \epsilon_{j}$, or

(b) $2 \mathbf{K} \delta^{\ell_{n}} \leqslant \epsilon_{j}^{\alpha}$.

In order to prove the claim let $\left\{P_{m}\right\}$ be a dyadic covering of $Q \cap E_{j}$ such that

$$
\sum_{m} \mu\left(P_{m}\right)^{t / d} \leqslant \mathcal{M}_{\mu, \infty}^{t}\left(Q \cap E_{j}\right)+\varepsilon .
$$

Next, let $p, q \in \mathbb{N}$ such that

$$
\begin{gathered}
2 \mathbf{K} \delta^{p} \leqslant \epsilon_{j}^{\alpha}<2 \mathbf{K} \delta^{p-1} \\
2 \mathbf{K} \delta^{q}<c_{1} \epsilon_{j} \leqslant 2 \mathbf{K} \delta^{q-1} .
\end{gathered}
$$

Suppose $P_{m}$ is such that $h_{m}=\operatorname{lev}\left(P_{m}\right)$ does not satisfies neither $(a)$ nor $(b)$ of the claim. Then we must have $q \leqslant h_{m}<p$ and $\operatorname{so} \operatorname{diam}\left(P_{m}\right) \leqslant 2 \mathbf{K} \delta^{h_{m}}<c_{1} \epsilon_{j}$. Since $\epsilon_{j}^{\alpha} \leqslant \frac{1}{2} c_{1} \epsilon_{j}$ and the points of the form $x_{k, j}$ are $2 c_{1} \epsilon_{j}$ separated, we have that $P_{m}$ intersects only one ball of $E_{j}$, which we denote by $B$. Let $P_{m, 1}, \ldots, P_{m, r_{m}}$ be the subcubes of $P_{m}$ of level $p$ that intersect $B$. By Lemma 2.3 we have that

$$
r_{m} \leqslant H\left(\epsilon_{j}^{\alpha} / \delta^{p}\right) \leqslant H(2 \mathbf{K} / \delta) .
$$

We define the covering $\left\{Q_{n}\right\}$ by changing all cubes $P_{m}$ as above by their respective subcubes $P_{m, 1}, \ldots, P_{m, r_{m}}$. Using that $\mu\left(P_{m, i}\right) \leqslant \mu\left(P_{m}\right)$ and (26) we get (25) and then the claim is proved.

Now consider $\left\{Q_{n}\right\}$ as in the claim. Observe also that we can suppose that $\delta^{\ell_{n}+1} \leqslant R$ for every $n$. We want to estimate $\nu_{j}\left(Q_{n}\right)$ for each $n$. For that we study separately the cases given by the claim. 
If $n$ satisfies $(a)$ we can use Lemma 5.2 to estimate for every $j \geqslant j_{0}$,

$$
\begin{aligned}
\nu_{j}\left(Q_{n}\right) \leqslant \frac{m_{j}\left(Q_{n}\right)}{n_{j}(Q)} & \leqslant \frac{C_{2} \epsilon_{j}^{-d}\left(\mathbf{K} \delta^{\ell_{n}}+2 c_{1} \epsilon_{j}\right)^{d}}{C_{2}^{-1} \epsilon_{j}^{-d}\left(R-\left(c_{1}+C_{1}\right) \epsilon_{j}\right)^{d}} \\
& \leqslant C_{2}^{2} \frac{\delta^{\ell_{n} t}}{R^{t}}\left(\frac{R^{t / d}\left(\mathbf{K} \delta^{\ell_{n}}+2 c_{1} \epsilon_{j}\right)}{\delta^{\ell_{n} t / d}\left(R-\left(c_{1}+C_{1}\right) \epsilon_{j}\right)}\right)^{d} \\
& \leqslant C_{2}^{2} \frac{\delta^{\ell_{n} t}}{R^{t}}\left(\frac{\mathbf{K} \delta^{\ell_{n}(1-t / d)} R^{t / d}}{\frac{1}{2} R}+\frac{2 c_{1} \epsilon_{j} R^{t / d} \delta^{-\ell_{n} t / d}}{\frac{1}{2} R}\right)^{d} \\
& \leqslant 2^{d} C_{2}^{2} \frac{\delta^{\ell_{n} t}}{R^{t}}\left(R^{t / d-1} \mathbf{K} \delta^{\ell_{n}(1-t / d)}+2^{1+t / d} R^{t / d-1} \mathbf{K}^{t / d}\left(c_{1} \epsilon_{j}\right)^{1-t / d}\right)^{d},
\end{aligned}
$$

by also using $\left(c_{1}+C_{1}\right) \epsilon_{j} \leqslant \frac{1}{2} R$ in the third line and $\delta^{-\ell_{n}} \leqslant 2 \mathbf{K}\left(c_{1} \epsilon_{j}\right)^{-1}$ in the last inequality. Since we also have $\delta^{\ell_{n}+1} \leqslant R$, we can deduce that for all $j \geqslant j_{0}$,

$$
\nu_{j}\left(Q_{n}\right) \leqslant 2^{d} C_{2}^{2} \frac{\delta^{\ell_{n} t}}{R^{t}}\left(\mathbf{K} \delta^{t / d-1}+2^{1+t / d} R^{(t / d-1)} \mathbf{K}^{t / d}\left(c_{1} \epsilon_{j}\right)^{1-t / d}\right)^{d} .
$$

If $n$ satisfies $(b)$, then $Q_{n}$ intersects at most one ball $B\left(x, \epsilon_{j}^{\alpha}\right)$ in $E_{j}$. We can use the that $X$ is Ahlfors-regular and Lemma 5.2 in order to obtain, for every $j \geqslant j_{0}$,

$$
\begin{aligned}
& \nu_{j}\left(Q_{n}\right) \leqslant \frac{\mu\left(Q_{n}\right)}{\mu\left(B\left(x, \epsilon_{j}^{\alpha}\right)\right) n_{j}(Q)} \leqslant \frac{\mathbf{A K}^{d} \delta^{\ell_{n} d}}{\mathbf{A}^{-1} \epsilon_{j}^{(\alpha-1) d} C_{2}^{-1}\left(R-\left(c_{1}+C_{1}\right) \epsilon_{j}\right)^{d}} \\
& \leqslant \mathbf{A}^{2} C_{2} \mathbf{K}^{t} \frac{\delta^{\ell_{n} t}}{R^{t}}\left(\frac{\epsilon_{j}^{1-\alpha}(2 \mathbf{K})^{1-t / d} \delta^{\ell_{n}(1-t / d)} R^{t / d}}{\frac{1}{2} R}\right)^{d} \\
& \leqslant 2^{d} \mathbf{A}^{2} C_{2} \mathbf{K}^{t} \frac{\delta^{\ell_{n} t}}{R^{t}}\left(\epsilon_{j}^{1-\alpha t / d} R^{t / d-1}\right)^{d}
\end{aligned}
$$

by using again $\left(c_{1}+C_{1}\right) \epsilon_{j} \leqslant \frac{1}{2} R$.

Now note that in any case, since $\alpha t<d$, then for every $\eta>0$ there exists $j_{1} \geqslant j_{0}$ such that if $j \geqslant j_{1}$,

$$
\nu_{j}\left(Q_{n}\right) \leqslant\left(C_{3}+\eta\right)^{d} \frac{\delta^{\ell_{n} t}}{R^{t}}
$$

holds for all $n$, where $C_{3}$ is a positive constant. Running the sum over all $n$ this yields

$$
1=\nu_{j}\left(Q \cap E_{j}\right) \leqslant \sum_{n} \nu_{j}\left(Q_{n}\right) \leqslant\left(C_{3}+\eta\right)^{d} R^{-t} \sum_{n} \delta^{\ell_{n} t} \leqslant \mathbf{A}\left(C_{3}+\eta\right)^{d} R^{-t} \sum_{n} \mu\left(Q_{n}\right)^{t / d},
$$

where in the last inequality we use that $\delta^{\ell_{n}+1} \leqslant \operatorname{diam}(X)$ and (12) eventually modifying the constant A. Therefore

$$
R^{t} \leqslant 2 \mathbf{A}\left(C_{3}+\eta\right)^{d} H(2 \mathbf{K} / \delta)\left(\mathcal{M}_{\mu, \infty}^{t}\left(Q \cap E_{j}\right)+\varepsilon\right) .
$$

Taking $\lim \sup$ when $j \rightarrow \infty$ we obtain

$$
\mathcal{M}_{\mu, \infty}^{t}(Q)=\mu(Q)^{t / d} \leqslant \mathbf{A} \mathbf{K}^{t} R^{t} \leqslant \mathbf{A}^{2} \mathbf{K}^{t} C_{3}^{d} H(2 \mathbf{K} / \delta)\left(\limsup _{j \rightarrow \infty} \mathcal{M}_{\mu, \infty}^{t}\left(Q \cap E_{j}\right)+\varepsilon\right)
$$

Since $\varepsilon$ is arbitrarily small, the proof finishes by putting $c=\left(\mathbf{A}^{2} \mathbf{K}^{t} C_{3}^{d} H(2 \mathbf{K} / \delta)\right)^{-1}$.

Before proceeding to prove Theorem 1.5 let us precise what we mean when we say that the sequence of positive real numbers $\mathcal{E}=\left\{\epsilon_{j}\right\}$ has exponential decay, this is:

$$
\limsup _{j \rightarrow \infty} \frac{\log \left(\epsilon_{j}\right)}{j}<0
$$


Proof of Theorem 1.5. Fix $t<d / \alpha$. By Lemma 5.1 there exists a constant $c \in(0,1)$ such that

$$
\limsup _{j \rightarrow \infty} \mathcal{M}_{\mu, \infty}^{t}\left(Q \cap E_{j}\right) \geqslant c \mathcal{M}_{\mu, \infty}^{t}(Q)
$$

holds for every dyadic cube $Q$. Therefore, for every $j$ and every dyadic cube $Q$ we have

$$
\mathcal{M}_{\mu, \infty}^{t}\left(\left(\bigcup_{j^{\prime} \geqslant j} E_{j^{\prime}}\right) \cup Q\right) \geqslant \sup _{j^{\prime} \geqslant j} \mathcal{M}_{\mu, \infty}^{t}\left(E_{j^{\prime}} \cap Q\right) \geqslant c \mathcal{M}_{\mu, \infty}^{t}(Q) .
$$

This means that $\bigcup_{j^{\prime} \geqslant j} E_{j^{\prime}}$ belongs to $\mathcal{J}_{\mu}^{t}$ for every $j$. Finally, (iii) of Theorem 1.3 allows us to conclude that $F=\bigcap_{j} \bigcup_{j^{\prime} \geqslant j} E_{j^{\prime}} \in \mathcal{J}_{\mu}^{t}$. Since $t<d / \alpha$ was arbitrary, we get that $F \in \mathcal{G}_{\mu}^{d / \alpha}$.

Now suppose that $\mathcal{E}$ has exponential decay and let $\beta>0$ be such that

$$
\limsup _{j \rightarrow \infty} \frac{\log \left(\epsilon_{j}\right)}{j}=-\beta \text {. }
$$

Thus there exists $j_{0}$ such that $\epsilon_{j} \leqslant e^{-\beta j / 2}$ for every $j \geqslant j_{0}$.

Now take $t>d / \alpha$. Consider an arbitrary ball $B=B\left(x_{0}, R\right) \subset X$ and let $0<r<R$. Let $j_{1} \geqslant j_{0}$ be such that $\epsilon_{j}^{\alpha} \leqslant c_{1} \epsilon_{j}$ and $2 \epsilon_{j}^{\alpha} \leqslant r$ for every $j \geqslant j_{1}$, and take the covering of $F \cap B$ that consists of all balls $B\left(x_{j, k}, \epsilon_{j}^{\alpha}\right)$ with $j \geqslant j_{1}$ that intersect $B$. Then applying the right hand side of (22) for $B$ and that $\epsilon_{j} \leqslant r \leqslant R$ we have

$$
\begin{aligned}
\mathcal{H}_{r}^{t}(F \cap B) & \leqslant \sum_{j \geqslant j_{1}} \sum_{k \in \mathcal{F}^{j}(B)} 2^{t} \epsilon_{j}^{\alpha t} \\
& \leqslant \sum_{j \geqslant j_{1}} m_{j}(B) 2^{t} \epsilon_{j}^{\alpha t} \leqslant 2^{t} C_{2}\left(1+2 c_{1}\right)^{d} R^{d} \sum_{j \geqslant j_{1}} \epsilon_{j}^{\alpha t-d}
\end{aligned}
$$

Since $\epsilon_{j} \leqslant e^{-\beta j / 2}$ and $t>d / \alpha$, the series $\sum \epsilon_{j}^{\alpha t-d}$ converges. Taking the limit when $j_{1} \rightarrow 0$ we see that $\mathcal{H}_{r}^{t}(F \cap B)=0$ and then $\mathcal{H}^{t}(F \cap B)=0$, which shows that $\mathcal{H}^{t}(F)=0$ since $B$ is any ball. Finally, as $t>d / \alpha$ is also arbitrary, we have that $\operatorname{dim}_{\mathcal{H}} F \leqslant d / \alpha$.

The estimate (27) shows that if $\sum \epsilon_{j}^{\alpha t-d}$ converges, then $\operatorname{dim}_{\mathcal{H}} F \leqslant t$. We can use this fact to get upper bounds for the dimension in the case of non-exponential decay. For example, if $\epsilon_{j}=j^{-t}$ with $t>0$, it is easy to see that $\operatorname{dim}_{\mathcal{H}} F \leqslant \frac{d t+1}{\alpha t}$.

\section{Comparison Between $\mathcal{J}_{\mu}^{s}, \mathcal{G}_{\mu}^{s}$ And other PREvious Definitions}

As we said in the introduction, although $\mathcal{G}^{s}\left(\mathbb{R}^{d}\right)=\mathcal{G}_{\mathcal{L}}^{s}\left(\mathbb{R}^{d}\right)$, for a general metric space $X$ characterizing $\mathcal{G}_{\mu}^{s}$-sets as in (1):

$$
\operatorname{dim}_{\mathcal{H}} \bigcap_{i} f_{i}^{-1}(F) \geqslant s
$$

where $f_{i}: V \rightarrow X$ are bi-Lipschitz maps defined on an open subset $V \subset X$, may not be the best fit since it is possible that $X$ does not have so many similarities or bi-Lipschitz maps to begin with and so the condition (28) might be weaker than that of Definition 1.1. Indeed, let us illustrate this in the following example, which lies in the Ahlfors regular context.

Example 6.1. Consider $X=H \cup L \subset \mathbb{R}^{3}$, where

$$
H=\left\{x=\left(x_{1}, x_{2}, x_{3}\right) \in \mathbb{R}^{3}: x_{3}=0\right\}, \quad L=\left\{x=\left(x_{1}, x_{2}, x_{3}\right) \in \mathbb{R}^{3}: x_{1}=x_{2}=0\right\} .
$$


We define the distance on $X$ by:

$$
|x-y|=\left\{\begin{array}{cc}
\|x-y\| & \text { if } x, y \in H \\
\|x-y\|^{1 / 2} & \text { if } x, y \in L \\
\|x\|+\|y\|^{1 / 2} & \text { if } x \in H, y \in L
\end{array}\right.
$$

where $\|\cdot\|$ denotes the usual Euclidean norm on $\mathbb{R}^{3}$. The metric space $X$ is thus obtained by gluing the Ahlfors 2-regular spaces $(H,\|\cdot\|)$ and $\left(L,\|\cdot\|^{1 / 2}\right)$, then it is Ahlfors 2-regular (see e.g. [20, Theorem 1.29]). We consider on $X$ the Hausdorff measure $\mu=\mathcal{H}^{2}$. By obvious topological obstructions there are not bi-Lipschitz maps that carry open sets from $H$ onto $L$, which allows us to take sets in $X$ satisfying condition (28) that are not dense in $X$.

Specifically let $F \subset L$ be a set of $\alpha$-approximable points with $\alpha=2 / s$. Applying Theorem 1.5 in $L$ we get that $F \in \mathcal{G}_{\mu}^{s}(L)$. Thus, by a combination of $(i)$ and $(i i)$ of Theorem 1.4 and $(i i i)$ of Theorem 1.3, $F$ also satisfies (28). But clearly $F \notin \mathcal{G}_{\mu}^{s}(X)$. Notice that this can be also written in terms of the $\mathcal{J}_{\mu}^{t}$-condition.

This example also shows that the class $\mathcal{G}_{\mu}^{s}(X)$ is not maximal among those classes of sets with dimension at least $s$ that are closed by countable intersections and bi-Lipschitz homeomorphisms. Indeed, the class $\mathcal{G}_{\mu}^{s}(X) \cup \mathcal{G}_{\mu}^{s}(L)$ is strictly larger and satisfies the same conditions.

Moreover, observe that with the same construction we can find two subsets $F, E \subset X$ that verify $(28)$ but with $\operatorname{dim}_{\mathcal{H}}(F \cap E)=0$, so the class defined by this condition is not closed under countable intersections.

The following example shows that the family $\mathcal{G}_{\mathcal{L}}^{s}\left(\mathbb{R}^{d}\right)$ is strictly larger than $\mathcal{J}_{\mathcal{L}}^{s}\left(\mathbb{R}^{d}\right)$. In order to simplify the notation, in what follows we write $\mathcal{G}^{s}=\mathcal{G}_{\mathcal{L}}^{s}\left(\mathbb{R}^{d}\right)$ and $\mathcal{J}^{s}=\mathcal{J}_{\mathcal{L}}^{s}\left(\mathbb{R}^{d}\right)$.

Example 6.2. Consider $X=\mathbb{R}^{d}$ with the euclidean distance and Lebesgue measure $\mathcal{L}$. Let $\alpha>1$ and set $s:=d / \alpha$. Define a sequence $\alpha_{j}=(1+1 / \sqrt{j}) \alpha$. Further, for each $j$ let $\left\{x_{j, k}\right\}_{k}$ be a $\left(1,2, e^{-j}\right)$-net and set

$$
E_{j}:=\bigcup_{k} B\left(x_{j, k}, e^{-j \alpha_{j}}\right), \quad F:=\lim \sup E_{j} .
$$

On the one hand, if $\beta>\alpha$, then the set of $\beta$-approximable points constructed from $\left\{x_{j, k}\right\}$ is contained in $F$. Then, by Theorem 1.5 for every $t=d / \beta<d / \alpha=s$ we have that $F \in \mathcal{G}^{t}$. Whence $F \in \mathcal{G}^{s}$.

On the other hand, for every ball $B=B\left(x_{0}, R\right) \subset \mathbb{R}^{d}$ we can compute $\mathcal{H}_{\infty}^{s}(F \cap B)$ as in the proof of Theorem 1.5. For a given $j_{1}$ we consider the covering of $F \cap B$ consisting of all balls of the form $B\left(x_{j, k}, e^{-j \alpha_{j}}\right)$ with $j \geqslant j_{1}$ that intersect $B$. Next, we take $j_{1}$ large enough so that $e^{-j \alpha_{j}} \leqslant e^{-j}<R$ for every $j \geqslant j_{1}$. Thus, using the notation of Lemma 5.2 , we see that the balls $B\left(x_{j, k}, e^{-j \alpha_{j}}\right)$ with $k \in \mathcal{F}^{j}(B)=\left\{k: B\left(x_{j, k}, e^{-j}\right) \cap B \neq 0\right\}$ and $j \geqslant j_{1}$ cover $F \cap B$. In sum

$$
\mathcal{H}_{\infty}^{s}(F \cap B) \leqslant \sum_{j \geqslant j_{1}} \sum_{k \in \mathcal{F}^{j}(B)}\left(2 e^{-j \alpha_{j}}\right)^{s} \leqslant 2^{s} \sum_{j \geqslant j_{1}} m_{j}(B) e^{-j(d+d / \sqrt{j})},
$$

where, as before, $m_{j}(B)=\# \mathcal{F}^{j}(B)$. Using the right hand side of $(22)$ and the fact that $e^{-j} \leqslant R$ for every $j \geqslant j_{1}$, we have that

$$
\mathcal{H}_{\infty}^{s}(F \cap B) \leqslant 2^{s} \sum_{j \geqslant j_{1}} C_{2} e^{j d}\left(R+2 e^{-j}\right) e^{-j(d+d / \sqrt{j})} \leqslant 2^{s} 3 C_{2} R \sum_{j \geqslant j_{1}} e^{-d \sqrt{j}} .
$$

Taking the limit when $j_{1} \rightarrow \infty$ we conclude that $\mathcal{H}_{\infty}^{s}(F \cap B)=0$, and thus, by $(i)$ of Theorem 1.4, $F \notin \mathcal{J}^{s}$. 
A third definition of these type of sets was given by Bugeaud in [6]. The idea is to generalize (4) considering a large class of dimension functions. A function $f:[0,+\infty) \rightarrow$ $[0,+\infty)$ is called a dimension function if it is strictly increasing, continuous and $f(0)=0$.

If $f$ is a dimension function we denote $\varepsilon(f)^{1}$ as the supremum of all positive numbers $x$ such that the function defined as $t \mapsto f\left(t^{1 / d}\right)$ is sub-additive on $[0, x]$. Then for a set $E \subset \mathbb{R}^{d}$ we define

$\mathcal{M}_{\infty}^{f}(E)=\inf \left\{\sum_{n} f\left(\operatorname{diam}\left(Q_{n}\right)\right): E \subset \bigcup_{n} Q_{n}, Q_{n}\right.$ dyadic cube with $\left.\operatorname{diam}\left(Q_{n}\right) \leqslant \varepsilon(f)\right\}$,

were, as in the introduction, we are considering the dyadic cubes in $\mathbb{R}^{d}$ as the family $Q_{j, k}=2^{j}\left(k+[0,1)^{d}\right), k \in \mathbb{Z}^{d}, j \in \mathbb{Z}$ (or $\mathbb{N}$ ). As before, $\mathcal{M}_{\infty}^{f}$ is equivalent to the Hausdorff content $\mathcal{H}_{\infty}^{f}$ defined similarly but with any type of covering. Subbaditivity plays again a key role in the identity

$$
\mathcal{M}_{\infty}^{f}(Q)=f(\operatorname{diam}(Q))
$$

for all cube $Q$ with $\operatorname{diam}(Q) \leqslant \varepsilon(f)$.

The last ingredient to define $\mathcal{G}^{f}$-sets is the relation of dimension functions: if $g$ is another dimension function we write $g \prec f$ when

$$
g(x) / f(x) \rightarrow+\infty
$$

monotonically when $x$ decreases to 0 on a neighborhood of 0 . Finally we say that a $G_{\delta}$ subset $F \subset \mathbb{R}^{d}$ is a $\mathcal{G}^{f}$-set if

$$
\mathcal{M}_{\infty}^{g}(F \cap Q)=\mathcal{M}_{\infty}^{g}(Q)
$$

holds for every dyadic cube $Q$ and every dimension function $g \prec f$. Equivalent characterizations as in Theorem 1.2 also exists for the class $\mathcal{G}^{f}$, see [6, Theorem 6].

One could naturally extend the definition of the class $\mathcal{G}^{f}$ to a metric space $X$ with a doubling measure $\mu$ by substituting $\operatorname{diam}\left(Q_{n}\right)$ by $\mu\left(Q_{n}\right)^{1 / d}$ where $d=\operatorname{dim}_{\mathcal{H}} X$. In a similar way we can consider $\mathcal{J}_{\mu}^{f}$-sets for $\mathbb{R}^{d}$ or more general metric spaces by changing (29) to the same identity for just $f$. In which case we have

$$
\mathcal{G}_{\mu}^{f}(X)=\bigcap_{g \prec f} \mathcal{J}_{\mu}^{g}(X) .
$$

Moreover, it can be shown [6, Lemma 2] that $\mathcal{J}^{f} \subset \mathcal{G}^{f}$. On the other hand, when we consider Falconer's definition and the dimension function $f(x)=x^{s}$ we have $\mathcal{G}^{x^{s}} \subset \mathcal{G}^{s}$ since $x^{t} \prec x^{s}$ whenever $t<s$. Altogether this means that

$$
\mathcal{J}^{s}=\mathcal{J}^{x^{s}} \subset \mathcal{G}^{x^{s}} \subset \mathcal{G}^{s}
$$

for $0<s \leqslant d$. And we know from Example 6.2 that $\mathcal{J}^{s} \varsubsetneqq \mathcal{G}^{s}$. We will now show that Bugeaud's definition coincides with our definition of $\mathcal{J}^{s}$ in $\mathbb{R}^{d}$.

Proposition 6.3. For all $0<s \leqslant d$ we have $\mathcal{G}^{x^{s}}=\mathcal{J}^{s}$.

Proof. Since $\mathcal{J}^{s} \subset \mathcal{G}^{x^{s}}$, we only need to prove the converse inclusion. To that end we will show that if $F \notin \mathcal{J}^{s}$, then there exists $g \prec x^{s}$ such that (29) is not verified for $g$ and $F$.

The condition $F \notin \mathcal{J}^{s}$ means that there exists a dyadic cube $Q$ and a constant $c<1$ for which we can find a dyadic covering $\left\{Q_{n}\right\}$ of $F \cap Q$ such that

$$
\sum_{n} \mathcal{L}\left(Q_{n}\right)^{s / d}<c \mathcal{L}(Q)^{s / d}
$$

where, as before, $\mathcal{L}$ denotes the Lebesgue measure in $\mathbb{R}^{d}$.

\footnotetext{
${ }^{1}$ Bugeaud's definition is slightly more demanding: $f$ must be concave in $[0, x]$ for all $x<\varepsilon(f)$. This, however, does not affect the definition or the results that follow, since the key property still is sub-additivity.
} 
Since $\operatorname{diam}(Q)=\sqrt{d} \mathcal{L}(Q)^{1 / d}$, then (30) translates to

$$
\sum_{n} \operatorname{diam}\left(Q_{n}\right)^{s}<c \operatorname{diam}(Q)^{s}
$$

From here we will proceed to construct a dimension function $g \prec x^{s}$. First, we denote $x_{n}=\operatorname{diam}\left(Q_{n}\right)$ and $a_{n}=x_{n}^{s}$. Eventually reordering the cubes we may assume that the sequence $\left\{a_{n}\right\}$ is decreasing. Remember that, according to the cubes we consider in $\mathbb{R}^{d}$, for all $n$ we must have $x_{n}=\sqrt{d} 2^{m_{n}}$ for some $m_{n} \in \mathbb{Z}$. Thus, in particular we have that, for all $n$, either $x_{n+1}=x_{n}$ or $x_{n+1} \leqslant x_{n} / 2$.

For every $n$ we set

$$
A_{n}=\sum_{m \geqslant n} a_{m}
$$

Then for every $k$ we consider $\nu(k)$ such that $A_{\nu(k)}<2^{-k}$ and $a_{\nu(k)}<a_{\nu(k)-1}$. The second condition implies $a_{\nu(k)} / a_{\nu(k)-1} \leqslant 2^{-s}$. We also take $k_{0}$ such that for every $k \geqslant k_{0}$,

$$
1+\frac{1}{1+k}<2^{s}
$$

and for every $r \in(0,1)$ we define the function $h_{r}: \mathbb{N} \rightarrow \mathbb{N}$ by

$$
h_{r}(k)=\left\{\begin{array}{cc}
1+r k_{0} & \text { if } k \leqslant k_{0}, \\
1+r k & \text { if } k>k_{0} .
\end{array}\right.
$$

Next, we construct a sequence $\left\{b_{n}\right\}$ in the following way: if $n \in[\nu(k), \nu(k+1))$, we set

$$
b_{n}:=h_{r}(k) a_{n} .
$$

Then we have $b_{n} / a_{n} \nearrow+\infty$ and

$$
\sum_{n} b_{n}=\sum_{k} \sum_{\nu(k)}^{\nu(k+1)-1} h_{r}(k) a_{n} \leqslant \sum_{k} h_{r}(k) A_{\nu(k)} \leqslant \sum_{k} h_{r}(k) 2^{-k}<+\infty .
$$

Also observe that either $b_{n+1} / b_{n}=a_{n+1} / a_{n} \leqslant 1$ or

$$
\frac{b_{n+1}}{b_{n}} \leqslant \frac{h_{r}(k) a_{n+1}}{h_{r}(k-1) a_{n}} \leqslant \frac{h_{r}(k) a_{\nu(k)}}{h_{r}(k-1) a_{\nu(k)-1}}<1,
$$

where the last inequality comes from the condition $a_{\nu(k)} / a_{\nu(k)-1} \leqslant 2^{-s}$ together with (31) and the fact that for every $k$ the function $r \mapsto r /(1+r k)$ is increasing. We conclude that $b_{n}$ is decreasing.

Now we define $H_{r}:(0,+\infty) \rightarrow(0,+\infty)$ such that:

- $H_{r}\left(x_{n}\right)=b_{n} / a_{n}$,

- $H_{r}$ is affine on each interval $\left[x_{n+1}, x_{n}\right]$, and

- $H_{r}(x)=H_{r}\left(x_{0}\right)$ for every $x \geqslant x_{0}$.

This function is clearly decreasing and satisfies

$$
\lim _{x \rightarrow 0} H_{r}(x)=+\infty .
$$

Finally we set $g_{r}:[0,+\infty) \rightarrow[0,+\infty)$ as

$$
g_{r}(x):= \begin{cases}H_{r}(x) x^{s} & \text { if } x>0 \\ 0 & \text { if } x=0 .\end{cases}
$$

We claim that $g_{r}$ is a dimension function. To see this it is enough to show that $g_{r}$ is increasing on every interval $\left[x_{n+1}, x_{n}\right]$. Indeed, since $g_{r}$ is continuous in $(0,+\infty), g_{r}\left(x_{n}\right)=$ $b_{n}$ and $b_{n} \searrow 0$, then, if $g_{r}$ is increasing on every interval $\left[x_{n+1}, x_{n}\right], g_{r}$ is global increasing and continuous at 0 . 
If $H_{r}\left(x_{n}\right)=H_{r}\left(x_{n+1}\right)$, then $g_{r}^{\prime}(x)=s H_{r}\left(x_{n}\right) x^{s-1}>0$ for every $x \in\left(x_{n+1}, x_{n}\right)$. In the other case $H_{r}\left(x_{n}\right)=h_{r}(k)<h_{r}(k+1)=H_{r}\left(x_{n+1}\right)$ with $k \geqslant k_{0}$, which implies

$$
H_{r}(x)=\left(\frac{-r}{x_{n}-x_{n+1}}\right) x+\frac{r x_{n}}{x_{n}-x_{n+1}}+1+r k, \quad \text { for every } x \in\left(x_{n+1}, x_{n}\right) .
$$

Then for every $x \in\left(x_{n+1}, x_{n}\right)$,

$$
\begin{aligned}
g_{r}^{\prime}(x)=H_{r}^{\prime}(x) x^{s}+s H_{r}(x) x^{s-1} & =x^{s-1}\left(\frac{-r x}{x_{n}-x_{n+1}}-\frac{s r x}{x_{n}-x_{n+1}}+\frac{s r x_{n}}{x_{n}-x_{n+1}}+s+s r k\right) \\
& >x^{s-1} r\left(\frac{-(1+s) x_{n}}{x_{n}-x_{n+1}}+s k\right) \geqslant x^{s-1} r(-2(1+s)+s k),
\end{aligned}
$$

where the last inequality comes from the relation $x_{n+1} \leqslant x_{n} / 2$. Thus, if we choose $k_{0} \geqslant 2(1+s) / s$, then $g_{r}^{\prime}(x)>0$ for every $x \in\left(x_{n+1}, x_{n}\right)$.

Finally note that, by $(32), g_{r}(x) \prec x^{s}$ and

$$
\sum_{n} g_{r}\left(\operatorname{diam}\left(Q_{n}\right)\right)=\sum_{n} g_{r}\left(x_{n}\right)=\sum_{n} b_{n}<+\infty .
$$

Moreover, since $g_{r}(x)$ decreases pointwise to $x^{s}$ when $r \searrow 0$, the Dominated Convergence Theorem implies that

$$
\lim _{r \rightarrow 0} \sum_{n} g_{r}\left(\operatorname{diam}\left(Q_{n}\right)\right)=\sum_{n} \operatorname{diam}\left(Q_{n}\right)^{s}<c \operatorname{diam}(Q)^{s}=c \lim _{r \rightarrow 0} g_{r}(\operatorname{diam}(Q)) .
$$

Which means that there exists $r_{0} \in(0,1)$ small enough such that if $g=g_{r_{0}}$, then

$$
\mathcal{M}_{\infty}^{g}(F \cap Q) \leqslant \sum_{n} g\left(\operatorname{diam}\left(Q_{n}\right)\right)<c g(\operatorname{diam}(Q))=c \mathcal{M}_{\infty}^{g}(Q),
$$

where the last equality comes from the sub-additivity of $g\left(x^{1 / d}\right)$ : as $\frac{g(x)}{x^{s}}$ is decreasing then given $0 \leqslant x \leqslant y$

$$
\begin{aligned}
g\left((x+y)^{1 / d}\right) \leqslant(x+y)^{s / d} \frac{g\left(y^{1 / d}\right)}{y^{s / d}} & \leqslant\left(x^{s / d}+y^{s / d}\right) \frac{g\left(y^{1 / d}\right)}{y^{s / d}} \\
& \leqslant g\left(y^{1 / d}\right)+x^{s / d} \frac{g\left(x^{1 / d}\right)}{x^{s / d}} \leqslant g\left(x^{1 / d}\right)+g\left(y^{1 / d}\right)
\end{aligned}
$$

also using the restriction $0<s \leqslant d$.

Anyhow, (33) shows that $F$ does not verify (29) for $g$ and $Q$ and therefore $F \notin \mathcal{G}^{x^{s}}$.

As a consequence of Example 6.2 and Proposition 6.3 we conclude that Falconer's and Bugeaud's definitions give different families. The key difference between them is the fact that the dimension functions $x \mapsto x^{t}$, with $t<s$, are not "dense enough" within the family of all dimension functions $g \prec x^{s}$. A more direct way to see this is by considering the following example.

Example 6.4. Fix $\alpha>1$ and for each $j$ let $\left\{x_{j, k}\right\}_{k}$ be a $\left(1,2, e^{-j}\right)$-net in $\mathbb{R}^{d}$. For every $j$ we define

$$
E_{j}=\bigcup_{k} B\left(x_{j, k}, \frac{e^{-\alpha j}}{j^{\kappa}}\right),
$$

where $\kappa>0$ is to be chosen later. Finally, we set $F:=\limsup _{j} E_{j}$. We observe that for every $\delta>\alpha$ there exists $j_{\delta, \kappa}$ such that $\frac{e^{-\alpha j}}{j^{\kappa}} \geqslant e^{-\delta j}$ for every $j \geqslant j_{\delta, \kappa}$, and thus $F$ contains a $\delta$-approximable set for every $\delta>\alpha$. Then, by Theorem 1.5, we have $F \in \mathcal{G}^{d / \delta}$ for every $\delta>\alpha$, which implies $F \in \mathcal{G}^{d / \alpha}$.

We consider the dimension functions $f(t)=t^{d / \alpha}$ and

$$
g(t)=t^{d / \alpha} \log \left(1+t^{-1}\right)^{\beta} .
$$


with $\beta>0$, which clearly satisfy $g \prec f$. Proceeding as in Example 6.2 and choosing $\kappa$ and $\beta$ so that $d \kappa / \alpha-\beta>1$ we obtain $\mathcal{H}_{\infty}^{g}(F)=0$, which implies $\mathcal{M}_{\infty}^{g}(F)=0$ and therefore $F \notin \mathcal{G}^{f}$.

In Examples 6.2 and 6.4 we have slightly modified the definion of $\alpha$-approximable sets in order to construct subsets that belong to certain classes and not to others. Let us use this idea once again to construct the following example, where a kind of $\mathcal{J}$-condition (or $\mathcal{G}$-condition) is satisfied locally.

Example 6.5. Consider $X=[2, \infty)$ and for every $j \in \mathbb{N}$ let $\left\{x_{j, k}\right\}_{k}$ be a family of $\left(1,2, e^{-j}\right)$-nets. Now we define for each $j$

$$
E_{j}:=\bigcup_{k} B\left(x_{j, k}, e^{-j x_{j, k}}\right),
$$

and then $F=\lim \sup E_{j}$.

For every closed interval $\mathcal{I}=[a, b] \subset X$ we denote $\mathcal{I}_{j}=\left\{k: x_{j, k} \in \mathcal{I}\right\}$ and can consider two subsets $F^{\mathcal{I}}=\lim \sup E_{j}^{\mathcal{I}}$ and $F_{\mathcal{I}}=\lim \sup E_{\mathcal{I}}^{j}$ with

$$
E_{j}^{\mathcal{I}}=\bigcup_{k \in \mathcal{I}_{j}} B\left(x_{j, k}, e^{-j a}\right) \text { and } E_{\mathcal{I}}^{j}=\bigcup_{k \in \mathcal{I}_{j}} B\left(x_{j, k}, e^{-j b}\right) .
$$

It is easy to see that $F_{\mathcal{I}} \subset F \cap I \subset F^{\mathcal{I}}$. Then $\frac{1}{b} \leqslant \operatorname{dim}_{\mathcal{H}} F \leqslant \frac{1}{a}$ because $F_{\mathcal{I}}$ and $F^{\mathcal{I}}$ are sets of $b$ and $a$-approximable points (respectively) and $\mathcal{E}$ has exponential decay. Since $F \cap \mathcal{I}$ has subsets of dimension arbitrarily close to $\frac{1}{a}$, we conclude that $\operatorname{dim}_{\mathcal{H}} F \cap \mathcal{I}=\frac{1}{a}$. The same argument shows that $\operatorname{dim}_{\mathcal{H}} F=\frac{1}{2}$. On the other hand, we have that $F \cap \mathcal{I} \in \mathcal{G}^{1 / b}(\mathcal{I})$.

This is a very irregular metric space. For example it is clear that its only self locally bi-Lipschitz map is the identity.

\section{ACKNowledgments}

Part of this work was done while both authors were working at Centro de Matemática in Universidad de la República, Uruguay. The first author was financed by a 3 month postdoctoral grant of the same institution.

The second author is supported by the Mathematical Center in Akademgorodok under the agreement No. 075-15-2019-1675 with the Ministry of Science and Higher Education of the Russian Federation.

\section{REFERENCES}

[1] R. Aimar, B. Iaffei, L. Nitti. On the Macias-Segovia metrization of quasi-metric spaces. Revista U. Mat. Argentina 41 (1998), 67-76.

[2] J. M. Aubry, D. Maman, S. Seuret. Local behavior of traces of Besov functions: Prevalent results. J. Func. Anal. 264 (2013), 631-660.

[3] A. Baker, W. Schmidt Diophantince Approximation and Hausdorff dimension. Proc. London. Math. Soc. 21 (2006), 1-11.

[4] V. Beresnevich, D. Dickinson, S.L. Velani. Measure theoretic laws for limsup sets. Mem. Amer. Math. Soc. 179 (2006).

[5] I. Borosh, S. Fraenkel. A generalization of Jarnik's theorem on diophantine approximations. Indag. Math. 75 (1972), 193-201.

[6] Y. Bugeaud. Intersective sets and diophantine approximation. Michigan Math. J. 52 (2004), 667-682.

[7] M. Christ. A Tb theorem with remarks on analytic capacity and the Cauchy integral. Colloq. Math. 60/61 (1990), 601-628.

[8] M. Dodson, B. Rynne, J. Vickers. Metric Diophantine approximation and Hausdorff dimension on manifolds. Math. Proc. Cambridge Philos. Soc. 105 (1989), 547-558.

[9] M. Dodson, B. Rynne, J. Vickers. Diophantine approximation and a lower bound for Hausdorff dimension Mathematika 37 (1990), 59-73.

[10] K. FALCOner. The geometry of fractal sets. University Press, Cambridge, 1985.

[11] K. FALCONER. Fractal Geometry: Mathematical Fundations and Applications. Wiley, 1990. 
[12] K. Falconer. Sets with large intersection properties. J. London Math. Soc. 49 (1994), 267-280.

[13] A. Fraysse, S. Jaffard. How smooth is almost every function in a Sobolev space? Rev. Mat. Ibero. 22 (2006), 663-683.

[14] D. Färm, T. Persson. Large intersection classes on fractals. Nonlinearity 24 (2011), 1291-1309.

[15] J. Heinonen. Lectures on analysis on metric spaces. Springer-Verlag New York, 2001.

[16] T. Hytönen, A. Kairema. Systems of dyadic cubes in a doubling metric space. Colloq. Math. 126 (2012), 1-33.

[17] S. JAFFARD. Old friends revisited: the multifractal nature of some classical functions. J. Fourier Anal. Appl. 3 (1997), 1-22.

[18] I. JARNík. Zur metrischen theories der Diophantischen approximationen. Pros. Mat. Fyz. 36 (1928), 91-106.

[19] R. Macías, C. Segovia. Lipschitz functions on spaces of homogeneous type. Adv. in Math. 33 (1979), $257-270$.

[20] G. Paulik. Gluing Spaces and Analysis. Bonn, 2004 (PhD Thesis).

[21] T. Persson. Inhomogeneous potentials, Hausdorff dimension and shrinking targets. Ann. H. Lebesgue 2 (2019), 1-37.

[22] C. A. Rogers. Hausdorff measures. University Press, Cambridge, 1970.

Departamento de Matemática y Estadística del Litoral, Universidad de la República, URUGUAY.

Email address: felipenegreira@adinet.com.uy

Sobolev Institute of Mathematics, 4 Acad. Koptyug Ave., Novosibirsk 630090, Russia

Email address: esequeiramanzino@gmail.com 